IJMMS 2004:5, 239-258

PII. S0161171204302085

http://ijmms.hindawi.com

(c) Hindawi Publishing Corp.

\title{
ON LINEAR DIFFERENCE EQUATIONS OVER RINGS AND MODULES
}

\author{
JAWAD Y. ABUHLAIL
}

Received 8 February 2003

\begin{abstract}
We develop a coalgebraic approach to the study of solutions of linear difference equations over modules and rings. Some known results about linearly recursive sequences over base fields are generalized to linearly (bi)recursive (bi)sequences of modules over arbitrary commutative ground rings.
\end{abstract}

2000 Mathematics Subject Classification: 16W30, 39A99.

1. Introduction. Although the theory of linear difference equations over base fields is well understood, the theory over arbitrary ground rings and modules is still under development. It is becoming more interesting and is gaining increasingly special importance mainly because of recent applications in coding theory and cryptography (e.g., $[10,15])$.

In a series of papers, Taft et al. (e.g., [17, 22, 24]) developed a coalgebraic aspect for the study of linearly recursive sequences over fields. Moreover, Grünenfelder et al. studied in $[8,9]$ the linearly recursive sequences over finite-dimensional vector spaces. Linearly recursive (bi)sequences over arbitrary rings and modules were studied intensively by Nechaev et al. (e.g., [16, 20, 21]); however, the coalgebraic approach in their work was limited to the field case. Generalization to the case of arbitrary commutative ground rings was studied by several authors including Kurakin [12, 13, 14] and eventually Abuhlail, Gómez-Torrecillas, and Wisbauer [4].

In this paper, we develop a coalgebraic aspect for the study of solutions of linear difference equations over arbitrary rings and modules. For some of our results, we assume that the ground ring is Artinian. Besides the new results, this paper extends results in [4] and "Kapitel 4" of my doctoral thesis [2]. A standard reference for the theory of linearly recursive sequences over rings and modules is the comprehensive work of Mikhalev et al. [16]. For the theory of Hopf algebras, the reader may refer to any of the classical references (e.g., [1, 19, 23]).

By $R$ we denote a commutative ring with $1_{R} \neq 0_{R}$ and with $U(R)=\{r \in R \mid r$ is invertible the group of units of $R$. The category of $R$-(bi)modules will be denoted by $M_{R}$. For an $R$-module $M$, we call an $R$-submodule $K \subset M$ pure (in the sense of Cohn) if for every $R$-module $N$, the induced map $\iota_{k} \otimes \operatorname{id}_{N}: K \otimes_{R} N \rightarrow M \otimes_{R} N$ is injective.

For an $R$-algebra $A$ and an $A$-module $M$, we call an $A$-submodule $K \subset M R$-cofinite if $M / K$ is finitely generated in $M_{R}$. For an $R$-algebra $A$, we denote by $\mathscr{K}_{A}$ the class of $R$-cofinite ideals. If $A$ is an $R$-algebra with $\mathscr{K}_{A}$ a filter, then we define for every left 
$A$-module $M$ the finite dual right $A$-module

$$
M^{\circ}:=\left\{f \in M^{*} \mid \operatorname{Ke}(f) \supset I M\right.
$$

for some $A$-ideal $I$ with $A / I$ finitely generated over $R\}$.

By $\mathbb{N}$ (resp., $\mathbb{Z}$ ) we denote the set of natural numbers (resp., the ring of integers). Moreover, we set $\mathbb{N}_{0}:=\{0,1,2,3, \ldots\}$. For an $n \times n$ matrix $M$ over $R$, we denote the characteristic polynomial by $\chi(M)$. The identity matrix of order $n$ over $R$ is denoted by $E_{n}$. For an $m \times n$ matrix $A$ and a $k \times l$ matrix $B$, the Kronecker product (tensor product) of $A$ and $B$ is the $m k \times n l$ matrix

$$
A \otimes B:=\left[\begin{array}{ccccc}
a_{11} \cdot B & a_{12} \cdot B & \ldots & \ldots & a_{1 n} \cdot B \\
a_{21} \cdot B & a_{22} \cdot B & \ldots & \ldots & a_{2 n} \cdot B \\
\vdots & \vdots & \vdots & \vdots & \vdots \\
a_{m 1} \cdot B & a_{m 2} \cdot B & \ldots & \cdots & a_{m n} \cdot B
\end{array}\right] .
$$

2. Preliminaries. Let $M$ be an $R$-module and

$$
M[\mathbf{x}]:=M\left[x_{1}, \ldots, x_{k}\right], \quad M\left[\mathbf{x}, \mathbf{x}^{-1}\right]:=M\left[x_{1}, x_{1}^{-1}, \ldots, x_{k}, x_{k}^{-1}\right] .
$$

We consider the polynomial ring $R[\mathbf{x}]$ and the ring of Laurent polynomials $R\left[\mathbf{x}, \mathbf{x}^{-1}\right]$ as commutative $R$-algebras with the usual multiplication and the usual unity. For every $R$ module $M, M[\mathbf{x}]$ (resp., $M\left[\mathbf{x}, \mathbf{x}^{-1}\right]$ ) is an $R[\mathbf{x}]$-module (resp., an $R\left[\mathbf{x}, \mathbf{x}^{-1}\right]$-module) with action induced from the $R$-module structure on $M$ and we have, moreover, canonical $R$-module isomorphisms

$$
M[\mathbf{x}] \simeq M \otimes_{R} R[\mathbf{x}] \simeq M^{\left(\mathbb{N}_{0}^{k}\right)}, \quad M\left[\mathbf{x}, \mathbf{x}^{-1}\right] \simeq M \otimes_{R} R\left[\mathbf{x}, \mathbf{x}^{-1}\right] \simeq M^{\left(\mathbb{Z}^{k}\right)} .
$$

For $\mathbf{n}=\left(n_{1}, \ldots, n_{k}\right) \in \mathbb{N}_{0}^{k}\left(\right.$ resp., $\left.\mathbf{z}=\left(z_{1}, \ldots, z_{k}\right) \in \mathbb{Z}^{k}\right)$, we set $\mathbf{x}^{\mathbf{n}}:=x_{1}^{n_{1}}, \ldots, x_{k}^{n_{k}}$ (resp., $\left.\mathbf{x}^{\mathbf{z}}:=x_{1}^{z_{1}}, \ldots, x_{k}^{z_{k}}\right)$.

2.1. Let $M$ be an $R$-module, $\mathbf{l}=\left(l_{1}, \ldots, l_{k}\right) \in \mathbb{N}_{0}^{k}$, and consider the system of linear difference equations (SLDE)

$$
\begin{gathered}
x_{\mathbf{n}+\left(l_{1}, 0, \ldots, 0\right)}+\sum_{i=1}^{l_{1}} p_{\left(1, l_{1}-i\right)}(\mathbf{n}) x_{\mathbf{n}+\left(l_{1}-i, 0, \ldots, 0\right)}=g_{1}(\mathbf{n}), \\
x_{\mathbf{n}+\left(0, l_{2}, 0, \ldots, 0\right)}+\sum_{i=1}^{l_{2}} p_{\left(2, l_{2}-i\right)}(\mathbf{n}) x_{\mathbf{n}+\left(0, l_{2}-i, 0, \ldots, 0\right)}=g_{2}(\mathbf{n}), \\
\vdots \\
x_{\mathbf{n}+\left(0, \ldots, 0, l_{k}\right)}+\sum_{i=1}^{l_{k}} p_{\left(k, l_{k}-i\right)}(\mathbf{n}) x_{\mathbf{n}+\left(0, \ldots, 0, l_{k}-i\right)}=g_{k}(\mathbf{n}),
\end{gathered}
$$

where the $p_{j l}$ 's are $R$-valued functions and the $g_{j}$ 's are $M$-valued functions defined for all $\mathbf{n} \in \mathbb{N}_{0}^{k}$. If the $g_{j}$ 's are identically zero, then (2.3) is said to be a homogenous SLDE. If the $p_{j l}$ 's are constants, then (2.3) is said to be an SLDE with constant coefficients. 
2.2. For an $R$-module $M$ and $k \geq 1$, let

$$
\mathscr{Y}_{M}^{\langle k\rangle}:=\left\{u: \mathbb{N}_{0}^{k} \longrightarrow M\right\} \simeq M^{\mathbb{N}_{0}^{k}}
$$

be the $R$-module of $k$-sequences over $M$. If $M$ (resp., $k$ ) is not mentioned, then we mean that $M=R$ (resp., $k=1$ ). For $f(\mathbf{x})=\sum_{\mathbf{i}} a_{\mathbf{i}} \mathbf{x}^{\mathbf{i}} \in R[\mathbf{x}]$ and $w \in \mathscr{Y}_{M}^{\langle k\rangle}$, define

$$
f(\mathbf{x})-w=u \in \mathscr{Y}_{M}^{\langle k\rangle}, \quad u(\mathbf{n}):=\sum_{\mathbf{i}} a_{\mathbf{i}} w(\mathbf{n}+\mathbf{i}) \forall \mathbf{n} \in \mathbb{N}_{0}^{k} .
$$

With this action, $\mathscr{S}_{M}^{\langle k\rangle}$ is an $R[\mathbf{x}]$-module. For subsets $I \subset R[\mathbf{x}]$ and $L \subset \mathscr{Y}_{M}^{\langle k\rangle}$, consider the annihilator submodules

$$
\begin{aligned}
& \operatorname{An}_{\mathscr{S}_{M}^{\langle k\rangle}}(I)=\left\{w \in \mathscr{S}_{M}^{\langle k\rangle} \mid f-w=0 \text { for every } f \in I\right\}, \\
& \operatorname{An}_{R[\mathbf{x}]}(L)=\{h \in R[\mathbf{x}] \mid h-u=0 \text { for every } u \in L\} .
\end{aligned}
$$

Note that $\operatorname{An}_{\varphi_{M}}^{\langle k\rangle}(I) \subset \mathscr{Y}_{M}^{\langle k\rangle}$ is an $R[\mathbf{x}]$-submodule and $\operatorname{An}_{R[\mathbf{x}]}(L) \triangleleft R[\mathbf{x}]$ is an ideal.

2.3. A polynomial $f(x) \in R[x]$ is called monic if its leading coefficient is $1_{R}$. For every monic polynomial $f(x)=x^{l}+a_{l-1} x^{l-1}+\cdots+a_{1} x+a_{0} \in R[x]$, the companion matrix of $f$ is defined to be the $l \times l$ matrix

$$
S_{f}:=\left[\begin{array}{ccccc}
0_{R} & 0_{R} & \cdots & 0_{R} & -a_{0} \\
1_{R} & 0_{R} & \cdots & 0_{R} & -a_{1} \\
0_{R} & 1_{R} & \cdots & 0_{R} & -a_{2} \\
\vdots & \vdots & \vdots & \vdots & \vdots \\
0_{R} & 0_{R} & \cdots & 1_{R} & -a_{l-1}
\end{array}\right] .
$$

In fact $S_{f}$ is a matrix that has $f(x)$ as its characteristic polynomial as well as its minimum polynomial (see [11, Theorem 4.18]).

DEFINITION 2.1. An ideal $I \triangleleft R[\mathbf{x}]$ will be called monic, if it contains a nonempty subset of monic polynomials

$$
\left\{f_{j}\left(x_{j}\right)=x_{j}^{l_{j}}+a_{l_{j}-1}^{(j)} x_{j}^{l_{j}-1}+\cdots+a_{1}^{(j)} x_{j}+a_{0}^{(j)} \mid j=1, \ldots, k\right\} .
$$

In this case, the polynomials (2.8) are called elementary polynomials and $\left(f_{1}\left(x_{1}\right), \ldots\right.$, $\left.f_{k}\left(x_{k}\right)\right) \triangleleft R[\mathbf{x}]$ an elementary ideal. A monic polynomial $q(x) \in R[x]$ is called reversible if $q(0) \in U(R)$. An ideal $I \triangleleft R\left[\mathbf{x}, \mathbf{x}^{-1}\right]$ will be called reversible if it contains a subset of reversible polynomials $\left\{q_{1}\left(x_{1}\right), \ldots, q_{k}\left(x_{k}\right)\right\}$.

2.4. Let $M$ be an $R$-module. We call $u \in \mathscr{Y}_{M}^{\langle k\rangle}$ a linearly recursive $k$-sequence (resp., a linearly birecursive $k$-sequence) if $\operatorname{An}_{R[\mathrm{x}]}(u)$ is a monic ideal (resp., a reversible ideal). Note that a $k$-sequence $u \in \mathscr{Y}_{M}^{\langle k\rangle}$ is linearly recursive if and only if it is a solution of a homogenous SLDE with constant coefficients of the form (2.3). If $\operatorname{An}_{R[\mathbf{x}]}(u)$ contains a set of monic polynomials $\left\{f_{1}\left(x_{1}\right), \ldots, f_{k}\left(x_{k}\right)\right\}$, where $f_{j}\left(x_{j}\right)$ is of order $m_{j}, j=1, \ldots, k$, then these are called elementary characteristic polynomials of $u$, and $u$ is said to have order $\mathbf{m}:=\left(m_{1}, \ldots, m_{k}\right)$. Characteristic polynomials of $u$ of least degree $n_{j}, j=1, \ldots, k$, 
are called minimal polynomials of $u$ and $\mathbf{n}:=\left(n_{1}, \ldots, n_{k}\right)$ is called the rank of $u$. The subsets $\mathscr{L}_{M}^{\langle k\rangle} \subseteq \mathscr{Y}_{M}^{\langle k\rangle}$ of linearly recursive $k$-sequences and $\mathscr{\Re}_{M}^{\langle k\rangle} \subseteq \mathscr{Y}_{M}^{\langle k\rangle}$ of linearly birecursive $k$-sequences are obviously $R[\mathbf{x}]$-submodules.

2.5. The lexicographical linear $\operatorname{order}(\preceq)$ on $\mathbb{N}_{0}^{k}$ is defined as follows: for $\mathbf{i}=\left(i_{1}, \ldots, i_{k}\right)$ and $\mathbf{n}=\left(n_{1}, \ldots, n_{k}\right) \in \mathbb{N}_{0}^{k}$, we say $\mathbf{i} \leq \mathbf{n}$ if the first number in the sequence of integers

$$
\left(n_{1}+\cdots+n_{k}\right)-\left(i_{1}+\cdots+i_{k}\right), \quad n_{1}-i_{1}, \ldots, n_{k}-i_{k}
$$

that is different from zero is positive (see [18, page 170]).

Let $M$ be an $R$-module, $\mathbf{F}:=\left\{f_{1}\left(x_{1}\right), \ldots, f_{k}\left(x_{k}\right)\right\} \subset R[\mathbf{x}]$ a subset of monic polynomials with $\operatorname{deg}\left(f_{j}\left(x_{j}\right)\right)=l_{j}$ for $j=1, \ldots, k, \mathbf{1}:=\left(l_{1}, \ldots, l_{k}\right), \mathbf{1}:=(1, \ldots, 1)$, and $I_{\mathrm{F}}:=\left(f_{1}, \ldots, f_{k}\right) \triangleleft$ $R[\mathbf{x}]$. Note that the natural order " $\leq$ " on $\mathbb{N}_{0}$ induces on $\mathbb{N}_{0}^{k}$ a partial order and we define the polyhedron $\Pi_{\mathrm{F}}=\Pi(\mathbf{l}):=\left\{\mathbf{i} \in \mathbb{N}_{0}^{k} \mid \mathbf{i} \leq \mathbf{l}-\mathbf{1}\right\}$. The initial polyhedron of values of $\omega \in \mathscr{Y}_{M}^{\langle k\rangle}$ is defined as $\omega\left(\Pi_{\mathrm{F}}\right):=\left\{\omega(\mathbf{i}) \mid \mathbf{i} \in \Pi_{\mathrm{F}}\right\}$. For $l=l_{1}, \ldots, l_{k}$, the points of the polyhedron $\Pi_{\mathrm{F}}$ build a chain $\mathbf{0}=\mathbf{i}_{0} \preceq \mathbf{i}_{1} \preceq \cdots \preceq \mathbf{i}_{l-1}$ and we can write $\omega\left(\Pi_{\mathrm{F}}\right)$ as an initial vector of values $\left(\omega(\mathbf{0}), \omega\left(\mathbf{i}_{1}\right), \ldots, \omega\left(\mathbf{i}_{l-1}\right)\right) \in M^{l}$.

Let $\omega \in \operatorname{An}_{\mathcal{S}_{M}^{(k)}}\left(f_{1}\left(x_{1}\right), \ldots, f_{k}\left(x_{k}\right)\right)$, where $f_{j}\left(x_{j}\right)$ is monic for $j=1, \ldots, n$ and write, for every $\mathbf{n}=\left(n_{1}, \ldots, n_{k}\right) \in \mathbb{N}_{0}^{k}$,

$$
x_{j}^{n_{j}}=h_{j}\left(x_{j}\right) f_{j}\left(x_{j}\right)+r_{j}\left(x_{j}\right), \quad \operatorname{deg}\left(r_{j}\left(x_{j}\right)\right)<l_{j} .
$$

If we set

$$
g^{(\mathbf{n})}(\mathbf{x}):=\prod_{j=1}^{k} r_{j}\left(x_{j}\right)=\sum_{\mathbf{i} \in \Pi_{\mathbf{F}}} a_{i}^{(\mathbf{n})} \mathbf{x}^{\mathbf{i}}, \quad v:=\mathbf{x}^{\mathbf{n}}-\omega=g^{(\mathbf{n})}(\mathbf{x})-\omega,
$$

then

$$
\omega(\mathbf{n})=v(\mathbf{0})=\sum_{\mathbf{i} \in \Pi_{\mathbf{F}}} a_{\mathbf{i}}^{(\mathbf{n})} \omega(\mathbf{i}) \quad \text { for every } \mathbf{n} \in \mathbb{N}_{0}^{k} .
$$

Consequently, $\omega$ is completely determined by the initial polyhedron of values $\omega\left(\Pi_{\mathrm{F}}\right)$. For $\mathbf{t} \in \Pi_{\mathrm{F}}$, define the sequence $e_{\mathbf{t}}^{\mathrm{F}} \in \operatorname{An}_{g_{R}^{\prime(j)}}\left(I_{\mathbf{F}}\right)$ with initial polyhedron of values $e_{\mathbf{t}}^{\mathrm{F}}(\mathbf{i})=$ $\delta_{\mathrm{i}, \mathrm{t}}$ for all $\mathbf{i} \in \Pi_{\mathrm{F}}$. The sequence $e_{1-1}^{\mathrm{F}}$ is called the impulse sequence of $\operatorname{An}_{\mathcal{f}_{R}^{(j)}}\left(I_{\mathrm{F}}\right)$.

3. Examples. We now give some examples of linearly recursive sequences. For more examples, the reader may refer to [16].

EXAMPLE 3.1 (geometric progression). Let $M$ be an $R$-module, $m \in M, r \in R$, and consider $w \in \mathscr{S}_{M}$ given by

$$
w(n):=r^{n} m \text { for every } n \in \mathbb{N}_{0} .
$$

Then $w \in \mathscr{L}_{M}$ with initial condition $w(0)=m$ and elementary characteristic polynomial $f(x)=x-r$. Moreover, $\operatorname{An}_{R[x]}(w)=R[x](x-r)+R[x] \operatorname{An}_{R}(r)$. 
EXAMPLE 3.2 (arithmetic progression). Let $M$ be an $R$-module, $\{p, q\} \subset M$, and consider $w \in \mathscr{Y}_{M}$ given by

$$
w(n):=p+n q \text { for every } n \in \mathbb{N}_{0} .
$$

Then $w \in \mathscr{L}_{M}$ with initial vector $(p, p+q)$ and elementary characteristic polynomial $f(x)=(x-1)^{2}$. If $\operatorname{An}_{R}(q)=0$, then $f(x)$ is a unique minimal polynomial of $w$. If $r \in \operatorname{An}_{R}(q)$, then $f_{r}(x)=(x-1)^{2}+r(x-1)$ is another minimal polynomial of $w$.

REMARK 3.3. An example of a nonrecursive sequence over $\mathbb{Z}$ is the sequence of prime positive numbers $\{2,3,5,7, \ldots\}$.

EXAMPLE 3.4. Let $E=\left\{f_{1}(x), \ldots, f_{k}(x)\right\} \subset R[x]$ be a subset of monic polynomials.

(1) Let $M$ be an $R$-module, $u_{i} \in \operatorname{An}_{\varphi_{M}}\left(f_{i}\right)$ for $i=1, \ldots, k$, and consider $u:=u_{1} \dot{+}$ $\cdots+u_{k} \in \mathscr{S}_{M}^{\langle k\rangle}$ defined by $u(\mathbf{n})=u_{1}\left(n_{1}\right)+\cdots+u_{k}\left(n_{k}\right)$. Then $u \in \operatorname{An}_{\mathscr{S}_{M}^{\langle k\rangle}}\left(g_{1}\left(x_{1}\right), \ldots\right.$, $g_{k}\left(x_{k}\right)$ ), where for $i=1, \ldots, k$,

$$
g_{i}\left(x_{i}\right)= \begin{cases}f_{i}\left(x_{i}\right), & f_{i}\left(1_{R}\right)=0_{R}, \\ f_{i}\left(x_{i}\right)\left(x_{i}-1_{R}\right), & \text { otherwise }\end{cases}
$$

(2) Let $M_{1}, \ldots, M_{k}$ be $R$-modules, $u_{i} \in \operatorname{An}_{\varphi_{M_{i}}}\left(f_{i}\right)$ for $i=1, \ldots, k, M:=M_{1} \oplus \cdots \oplus M_{k}$, and consider $u \in \mathscr{S}_{M}^{\langle k\rangle}$ defined by $u(\mathbf{n}):=\left(u_{1}\left(n_{1}\right), \ldots, u_{k}\left(n_{k}\right)\right)$. Then $u \in \operatorname{An}_{\mathscr{S}_{M}^{\langle k\rangle}}\left(g_{1}\left(x_{1}\right)\right.$, $\ldots, g_{k}\left(x_{k}\right)$ ), where the $g_{i}$ 's are defined as in (3.3).

(3) Let $u_{i} \in \operatorname{An}_{\varphi_{R}}\left(f_{i}\right)$ for $i=1, \ldots, k$ and consider $u \in \mathscr{Y}_{R}^{\langle k\rangle}$ defined by $u(\mathbf{n}):=$ $u_{1}\left(n_{1}\right), \ldots, u_{k}\left(n_{k}\right)$. Then $u \in \operatorname{An}_{\mathscr{S}_{R}^{\langle k\rangle}}\left(f_{1}\left(x_{1}\right), \ldots, f_{k}\left(x_{k}\right)\right)$ and

$$
\operatorname{An}_{\mathscr{S}_{R}^{\langle k\rangle}}\left(f_{1}\left(x_{1}\right), \ldots, f_{k}\left(x_{k}\right)\right) \simeq \operatorname{An}_{\varphi_{R}}\left(f_{1}\right) \otimes_{R} \cdots \otimes_{R} \operatorname{An}_{\varphi_{R}}\left(f_{k}\right)
$$

(4) Let $M_{1}, \ldots, M_{k}$ be $R$-modules, $u_{i} \in \operatorname{An}_{\varphi_{M_{i}}}\left(f_{i}\right)$ for $i=1, \ldots, k, M:=M_{1} \otimes_{R} \cdots \otimes_{R} M_{k}$, and consider $u \in \mathscr{Y}_{M}^{\langle k\rangle}$ defined by $u(\mathbf{n}):=u_{1}\left(n_{1}\right) \otimes \cdots \otimes u_{k}\left(n_{k}\right)$. Then $u \in \operatorname{An}_{\mathscr{S}_{M}^{\langle k\rangle}}\left(f_{1}\left(x_{1}\right)\right.$, $\left.\ldots, f_{k}\left(x_{k}\right)\right)$ and

$$
\operatorname{An}_{\mathscr{S}_{M}^{\langle k\rangle}}\left(f_{1}\left(x_{1}\right), \ldots, f_{k}\left(x_{k}\right)\right) \simeq \operatorname{An}_{\varphi_{M_{1}}}\left(f_{1}\right) \otimes_{R} \cdots \otimes_{R} \operatorname{An}_{\varphi_{M_{k}}}\left(f_{k}\right)
$$

4. Admissible $R$-bialgebras and Hopf $R$-algebras. For every $R$-coalgebra $\left(C, \Delta_{C}, \varepsilon_{C}\right)$, there is a dual $R$-algebra $C^{*}:=\operatorname{Hom}_{R}(C, R)$ with the so-called convolution product multiplication

$$
(f * g)(c):=\sum f\left(c_{1}\right) g\left(c_{2}\right) \quad \forall f, g \in C^{*}, c \in C,
$$

and unity $\varepsilon_{C}$. Although every algebra $A$ has a dual coalgebra, if the ground ring is hereditary Noetherian (e.g., a field), the existence of dual coalgebras of algebras over an arbitrary commutative ground ring is not guaranteed! One way to handle this problem is to restrict the class of $R$-algebras for which the dual $R$-coalgebras are defined. 
Definition 4.1. Let $A$ be an $R$-algebra (resp., an $R$-bialgebra, a Hopf $R$-algebra). Then $A$ is called

(1) an $\alpha$-algebra (resp., an $\alpha$-bialgebra, a Hopf $\alpha$-algebra) if $\mathscr{K}_{A}$ is a filter and $A^{\circ} \subset R^{A}$ is pure;

(2) cofinitary if $\mathscr{K}_{A}$ is a filter and, for every $I \in \mathscr{K}_{A}$, there exists an $A$-ideal $\bar{I} \subseteq I$ with $A / \bar{I}$ finitely generated and projective.

4.1. Let $H$ be an $R$-bialgebra and consider the class of $R$-cofinite $H$-ideals $\mathscr{K}_{H}$. We call $H$ an admissible $R$-bialgebra if $H$ is cofinitary and $\mathscr{K}_{H}$ satisfies the following axioms:

(A1) for all $I, J \in \mathscr{K}_{H}$, there exists $L \in \mathscr{K}_{H}$, such that $\Delta_{H}(L) \subseteq \operatorname{Im}\left(I \otimes_{R} H\right)+\operatorname{Im}\left(H \otimes_{R} J\right)$, (A2) there exists $I \in \mathscr{K}_{H}$, such that $\operatorname{Ke}\left(\varepsilon_{H}\right) \supset I$.

We call a Hopf $R$-algebra $H$ an admissible Hopf $R$-algebra if $H$ is cofinitary and $\mathscr{K}_{H}$ satisfies (A1), (A2), and

(A3) for every $I \in \mathscr{K}_{H}$, there exists $J \in \mathscr{K}_{H}$, such that $S_{H}(J) \subseteq I$.

REMARK 4.2. It follows from the proof of [3, Proposition 4.2.] that every cofinitary $R$-algebra (resp., $R$-bialgebra, Hopf $R$-algebra) is an $\alpha$-algebra (resp., an $\alpha$-bialgebra, a Hopf $\alpha$-algebra). By [2, Lemma 2.5.6.], every cofinitary bialgebra (Hopf algebra) over a Noetherian ground ring is admissible.

Proposition 4.3 (see [2, Propositions 2.4.13 and 2.5.7]). (1) If $A$ is a cofinitary $R$ algebra, then $A^{\circ}$ is an $R$-coalgebra. If $H$ is an admissible $R$-bialgebra (resp., an admissible Hopf $R$-algebra), then $H^{\circ}$ is an $R$-bialgebra (resp., a Hopf $R$-algebra).

(2) Let $R$ be Noetherian. If $A$ is an $\alpha$-algebra (resp., an $\alpha$-bialgebra, a Hopf $\alpha$-algebra), then $A^{\circ}$ is an $R$-coalgebra (resp., an $R$-bialgebra, a Hopf $R$-algebra).

Proposition 4.4. Let $A$ be an $\alpha$-algebra (resp., an $\alpha$-bialgebra, a Hopf $\alpha$-algebra), $B$ a cofinitary $R$-algebra (resp., $R$-bialgebra, Hopf $R$-algebra), and consider the canonical map $\sigma: A^{\circ} \otimes_{R} B^{\circ} \rightarrow\left(A \otimes_{R} B\right)^{\circ}$. Then

(1) $\sigma$ is injective,

(2) if $R$ is Noetherian, then $\sigma$ is an isomorphism of $R$-coalgebras (resp., $R$-bialgebras, Hopf R-algebras).

Proof. (1) The proof is along the lines of the proof of [14, Proposition 5].

(2) The proof is along the lines of the proof of [3, Theorem 4.10].

The proof of [3, Lemma 4.12] can be generalized to prove the following lemma.

LEMMA 4.5. For any set of reversible polynomials $\left\{q_{1}\left(x_{1}\right), \ldots, q_{k}\left(x_{k}\right)\right\} \subseteq R[\mathbf{x}]$, there is an isomorphism of $R$-algebras

$$
R[\mathbf{x}] /\left(q_{1}\left(x_{1}\right), \ldots, q_{k}\left(x_{k}\right)\right) \simeq R\left[\mathbf{x}, \mathbf{x}^{-1}\right] /\left(q_{1}\left(x_{1}\right), \ldots, q_{k}\left(x_{k}\right)\right) .
$$

LEMMA 4.6 (see [14, Proposition 1]). Let $R$ be an arbitrary commutative ring.

(1) An ideal $I \triangleleft R[\mathbf{x}]$ is $R$-cofinite if and only if it is monic. Consequently, every $R$ cofinite $R[\mathbf{x}]$-ideal contains an ideal $\bar{I} \triangleleft R[\mathbf{x}]$ such that $R[\mathbf{x}] / \bar{I}$ is free of finite rank. In particular, $R[\mathbf{x}]$ is cofinitary. 
(2) An ideal $I \triangleleft R\left[\mathbf{x}, \mathbf{x}^{-1}\right]$ is $R$-cofinite if and only if it is reversible. Consequently, every $R$-cofinite $R\left[\mathbf{x}, \mathbf{x}^{-1}\right]$-ideal contains an ideal $\bar{I} \triangleleft R\left[\mathbf{x}, \mathbf{x}^{-1}\right]$ such that $R\left[\mathbf{x}, \mathbf{x}^{-1}\right] / \bar{I}$ is free of finite rank. In particular, $R\left[\mathbf{x}, \mathbf{x}^{-1}\right]$ is cofinitary.

5. Linearly (bi)recursive sequences. In this section, we study the linearly (bi)recursive $k$-sequences over $R$-modules, where $R$ is an arbitrary commutative ground ring.

5.1. Let $\left(G, \mu_{G}, e_{G}\right)$ be a (commutative) monoid. Considering the elements of the basis $G$ as group-like elements, the monoid algebra $R G$ becomes a (commutative) cocommutative $R$-bialgebra $\left(R G, \mu, \eta, \Delta_{g}, \varepsilon_{g}\right)$, where

$$
\Delta_{g}(x)=x \otimes x, \quad \varepsilon_{g}(x)=1_{R} \quad \text { for every } x \in G .
$$

If $G$ is a group, then $R G$ is a Hopf $R$-algebra with antipode

$$
S_{g}: R G \longrightarrow R G, \quad x \longmapsto x^{-1} \text { for every } x \in G \text {. }
$$

5.2. Bialgebra structures on $R[\mathbf{x}]$. Consider the commutative monoid $G$ generated by $\left\{x_{j} \mid j=1, \ldots, k\right\}$. Then $R[\mathbf{x}]=R G$ has the structure of a commutative cocommutative $R$-bialgebra $R[\mathbf{x} ; g]=\left(R[\mathbf{x}], \mu, \eta, \Delta_{g}, \varepsilon_{g}\right)$, where $\mu$ is the usual multiplication, $\eta$ is the usual unity, and for all $n \geq 0, j=1, \ldots, k$,

$$
\begin{gathered}
\Delta_{g}: R[\mathbf{x}] \longrightarrow R[\mathbf{x}] \otimes_{R} R[\mathbf{x}], \quad x_{j}^{n} \longmapsto x_{j}^{n} \otimes x_{j}^{n}, \\
\varepsilon_{g}: R[\mathbf{x}] \longrightarrow R, \quad x_{j}^{n} \longmapsto 1_{R} .
\end{gathered}
$$

On the other hand, $R[\mathbf{x} ; p]=\left(R[\mathbf{x}], \mu, \eta, \Delta_{p}, \varepsilon_{p}\right)$ is a commutative cocommutative Hopf $R$-algebra, where $\mu$ is the usual multiplication, $\eta$ is the usual unity, and for all $n \geq 0$, $j=1, \ldots, k$,

$$
\begin{aligned}
& \Delta_{p}: R[\mathbf{x}] \longrightarrow R[\mathbf{x}] \otimes_{R} R[\mathbf{x}], x_{j}^{n} \longmapsto \sum_{t=0}^{n}\left(\begin{array}{l}
n \\
t
\end{array}\right) x_{j}^{t} \otimes x_{j}^{n-t}, \\
& \varepsilon_{p}: R[\mathbf{x}] \longrightarrow R, x_{j}^{n} \longmapsto \delta_{n, 0}, \\
& S_{p}: R[\mathbf{x}] \longrightarrow R[\mathbf{x}], \quad x_{j}^{n} \longmapsto(-1)^{n} x_{j}^{n} .
\end{aligned}
$$

REMARKS 5.1. (1) Let $R$ be an integral domain, then it follows by [7, Theorem 1.3.6] that for every set $G$, the class of group-like elements of the $R$-coalgebra $R G$ is $G$ itself. Then one can show, as in the field case [6], that $R[\mathbf{x} ; g]$ and $R[\mathbf{x} ; p]$ are the only possible $R$-bialgebra structures on $R[\mathbf{x}]$ with the usual multiplication and the usual unity.

(2) The $R$-bialgebra $R[\mathbf{x} ; g]$ has no antipode because the group-like elements in a Hopf $R$-algebra should be invertible.

The proof of the following result depends mainly on the arguments of [14, Theorem 2].

Proposition 5.2. Let $R$ be an arbitrary commutative ring. Then $R[\mathbf{x} ; g]$ is an admissible $R$-bialgebra and $R[\mathbf{x} ; p]$ is an admissible Hopf $R$-algebra. Hence, $R[\mathbf{x} ; g]^{\circ}$ is an $R$-bialgebra and $R[\mathbf{x} ; p]^{\circ}$ is a Hopf R-algebra. 
Proof. Denote by $(R[\mathbf{x}], \Delta, \varepsilon)$ either of the cofinitary $R$-bialgebras $R[\mathbf{x} ; g]$ and $R[\mathbf{x} ; p]$. Let $I, J \triangleleft R[\mathbf{x}]$ be $R$-cofinite ideals and assume without loss of generality that $R[\mathbf{x}] / I$ and $R[\mathbf{x}] / J$ are free of finite rank (see Lemma 4.6). Let $\beta$ be a basis of the free $R$-module $B:=R[\mathbf{x}] / I \otimes_{R} R[\mathbf{x}] / J$ and consider the $R$-algebra morphism $\bar{\Delta}:=\left(\pi_{I} \otimes \pi_{J}\right) \circ \Delta: R[\mathbf{x}] \rightarrow$ $R[\mathbf{x}] / I \otimes_{R} R[\mathbf{x}] / J$. For $j=1, \ldots, k$, let $M_{j}$ be the matrix of the $R$-linear map

$$
T_{j}: B \longrightarrow B, \quad b \longmapsto \bar{\Delta}\left(x_{j}\right) b
$$

with respect to $\beta$, and $\chi_{j}(\lambda)$ its characteristic polynomial. Then $\chi_{j}\left(\bar{\Delta}\left(x_{j}\right)\right)=0$ for $j=$ $1, \ldots, k$. Since $\bar{\Delta}$ is an $R$-algebra morphism, it follows that $\chi_{j}\left(x_{j}\right) \in \operatorname{Ke}(\bar{\Delta})=\Delta^{-1}\left(I \otimes_{R}\right.$ $\left.R[\mathbf{x}]+R[\mathbf{x}] \otimes_{R} J\right)$ for $j=1, \ldots, k$. If we set $L:=\left(\chi_{1}\left(x_{1}\right), \ldots, \chi_{k}\left(x_{k}\right)\right) \triangleleft R[\mathbf{x}]$, then $\Delta(L) \subseteq$ $I \otimes_{R} R[\mathbf{x}]+R[\mathbf{x}] \otimes_{R} J$, that is, $\mathscr{K}_{R[\mathbf{x}]}$ satisfies axiom (A1). Note that $R[\mathbf{x}] / \operatorname{Ke}(\varepsilon) \simeq R$, hence $\mathscr{K}_{R[\mathbf{x}]}$ satisfies axiom (A2). Consequently, $R[\mathbf{x} ; g]$ and $R[\mathbf{x} ; p]$ are admissible $R$ bialgebras. Consider now the Hopf $R$-algebra $R[\mathbf{x} ; p]$ with the bijective antipode $S_{p}$. For every ideal $I \triangleleft R[\mathbf{x}], S_{p}^{-1}(I) \triangleleft R[\mathbf{x} ; p]$ is an ideal and we have an isomorphism of $R$-modules $R[\mathbf{x}] / S_{p}^{-1}(I) \simeq R[\mathbf{x}] / I$, hence $\mathscr{K}_{R[\mathbf{x} ; p]}$ satisfies axiom (A3). Consequently, $R[\mathbf{x} ; p]$ is an admissible Hopf $R$-algebra. The last statement follows now by Proposition 4.3 .

If $M$ is an arbitrary $R$-module, then we have obviously an isomorphism of $R[\mathbf{x}]$ modules

$$
\Phi_{M}: M[\mathbf{x}]^{*} \longrightarrow \mathscr{G}_{M^{*}}^{\langle k\rangle}, \quad \varkappa \longmapsto\left[\mathbf{n} \longmapsto\left[m \longmapsto \varkappa\left(m \mathbf{x}^{\mathbf{n}}\right)\right]\right]
$$

with inverse $u \mapsto\left[m \mathbf{x}^{\mathbf{n}} \mapsto u(\mathbf{n})(m)\right]$.

Proposition 5.3. Let $M$ be an $R$-module. Then (5.6) induces an isomorphism of $R[\mathbf{x}]$ modules

$$
M[\mathbf{x}]^{\circ} \simeq \mathscr{L}_{M^{*}}^{\langle k\rangle}
$$

Proof. Consider the $R[\mathbf{x}]$-module isomorphism $M[\mathbf{x}] \stackrel{\Phi_{M}}{\simeq} \mathscr{S}_{M^{*}}^{\langle k\rangle}$, see (5.6). Let $\varkappa \in$ $M[\mathbf{x}]^{\circ}$. Then there exists an $R$-cofinite $R[\mathbf{x}]$-ideal $I$ such that $I \rightarrow \varkappa=0$. So $I \rightarrow \Phi_{M}(\varkappa)=$ $\Phi_{M}(I-\varkappa)=0$, that is, $I \subset \operatorname{An}_{R[\mathrm{x}]}\left(\Phi_{M}(\varkappa)\right)$. By Lemma 4.6(1), $I$ is monic, that is, $\Phi_{M}(\varkappa) \in$ $\mathscr{L}_{M^{*}}^{\langle k\rangle}$

On the other hand, let $u \in \mathscr{L}_{M^{*}}^{\langle k\rangle}$. By definition, $J:=\operatorname{An}_{R[\mathbf{x}]}(u)$ is a monic ideal and it follows by Lemma 4.6(1) that $J \triangleleft R[\mathbf{x}]$ is $R$-cofinite. For $\varkappa:=\Phi_{M}^{-1}(u)$, we have $J \rightarrow \varkappa=$ $J \rightarrow \Phi_{M}^{-1}(u)=\Phi_{M}^{-1}(J-u)=0$, that is, $\varkappa \in M[\mathbf{x}]^{\circ}$.

5.3. The coalgebra structure on $\mathscr{L}^{\langle k\rangle}$. By Lemma $4.6(1),(R[\mathbf{x}], \mu, \eta)$ is a cofinitary $R$ algebra, where $\mu$ is the usual multiplication and $\eta$ is the usual unity. Hence, $\left(R[\mathbf{x}]^{\circ}, \mu^{\circ}\right.$, $\eta^{\circ}$ ) is (by Proposition 4.3) an $R$-coalgebra, where

$$
\begin{aligned}
\mu^{\circ}: R[\mathbf{x}]^{\circ} \longrightarrow R[\mathbf{x}]^{\circ} \otimes_{R} R[\mathbf{x}]^{\circ}, \quad f & \longmapsto\left[x_{i}^{s} \otimes x_{j}^{t} \longmapsto f\left(x_{i}^{s} x_{j}^{t}\right), \quad s, t \geq 0, i, j=1, \ldots, k\right], \\
\eta^{\circ}: R[\mathbf{x}]^{\circ} & \longrightarrow R, \quad f \longmapsto f\left(1_{R}\right) .
\end{aligned}
$$


So $\mathscr{L}^{\langle k\rangle} \simeq R[\mathbf{x}]^{\circ}$ has the structure of an $R$-coalgebra with counity

$$
\varepsilon_{\mathscr{L}}\langle k\rangle: \mathscr{L}^{\langle k\rangle} \longrightarrow R, \quad u \longmapsto u(\mathbf{0}),
$$

and comultiplication described as follows (see [16, Proposition 14.16]).

Let $u \in \mathscr{L}^{\langle k\rangle},\left\{f_{1}\left(x_{1}\right), \ldots, f_{k}\left(x_{k}\right)\right\} \subseteq \mathrm{An}_{R[\mathrm{x}]}(u)$ a subset of elementary characteristic polynomials with $\operatorname{deg}\left(f_{j}\left(x_{j}\right)\right)=l_{j}$, and $\mathbf{l}:=\left(l_{1}, \ldots, l_{k}\right)$. So we have for all $\mathbf{n}, \mathbf{i} \in \mathbb{N}_{0}^{k}$,

$$
u(\mathbf{n}+\mathbf{i})=\left(\mathbf{x}^{\mathbf{i}}-u\right)(\mathbf{n})=\left(\sum_{\mathbf{t} \leq \mathbf{l}-\mathbf{1}}\left(\mathbf{x}^{\mathbf{i}}-u\right)(\mathbf{t}) \cdot e_{\mathbf{t}}^{\mathbf{F}}\right)(\mathbf{n})=\sum_{\mathbf{t} \leq \mathbf{l}-\mathbf{1}}\left(\mathbf{x}^{\mathbf{t}}-u\right)(\mathbf{i}) \cdot e_{\mathbf{t}}^{\mathbf{F}}(\mathbf{n}) .
$$

The comultiplication of $\mathscr{L}^{\langle k\rangle}$ is given then by

$$
\Delta_{\mathscr{L}\langle k\rangle}: \mathscr{L}^{\langle k\rangle} \longrightarrow \mathscr{L}^{\langle k\rangle} \otimes_{R} \mathscr{L}^{\langle k\rangle}, \quad u \longmapsto \sum_{\mathbf{t} \leq \mathbf{l}-\mathbf{1}}\left(\mathbf{x}^{\mathbf{t}}-u\right) \otimes e_{\mathbf{t}}^{\mathbf{F}}
$$

EXAMPLE 5.4. Consider the Fibonacci sequence $\digamma=(0,1,1,2,3,5, \ldots)$. Clearly, $\digamma$ is given by

$$
\digamma(0)=0, \quad \digamma(1)=1, \quad \digamma(n+2)=\digamma(n+1)+\digamma(n) \quad \forall n \geq 0,
$$

that is, $\digamma \in \mathscr{L}_{\mathbb{Z}}$ with initial vector $(0,1)$ and elementary characteristic polynomial $f(x)=$ $x^{2}-x-1 \in \mathbb{Z}[x]$. By (5.11), one can easily calculate

$$
\Delta_{\mathscr{L}_{\mathbb{Z}}}(\digamma)=\digamma \otimes_{\mathbb{Z}}(x-\digamma)+(x-\digamma) \otimes_{\mathbb{Z}} \digamma-\digamma \otimes_{\mathbb{Z}} \digamma .
$$

5.4. The $R$-bialgebra $\left(\mathscr{L}_{R}^{\langle k\rangle} ; g\right)$. Consider the $R$-bialgebra $R[\mathbf{x} ; g]$. Then $\mathscr{S}^{\langle k\rangle} \simeq R^{\mathbb{N}_{0}^{k}} \simeq$ $R[\mathbf{x} ; g]^{*}$ is an $R$-algebra with multiplication given by the Hadamard product

$$
*_{g}: \mathscr{\varphi}^{\langle k\rangle} \otimes_{R} \mathscr{\varphi}^{\langle k\rangle} \longrightarrow \mathscr{\varphi}^{\langle k\rangle}, \quad u \otimes v \longmapsto[\mathbf{n} \longmapsto u(\mathbf{n}) v(\mathbf{n})]
$$

and the unity

$$
\eta_{g}: R \longrightarrow \mathscr{Y}^{\langle k\rangle}, \quad 1_{R} \longmapsto\left[\mathbf{n} \longmapsto 1_{R}\right] \text { for every } \mathbf{n} \in \mathbb{N}_{0}^{k}
$$

By Propositions 5.2 and $5.3,\left(\mathscr{L}_{R}^{\langle k\rangle} ; g\right) \simeq R[\mathbf{x} ; g]^{\circ}$ has the structure of an $R$-bialgebra with the coalgebra structure described in Section 5.3, the Hadamard product (5.14), and the unity (5.15).

5.5. The Hopf $R$-algebra $\left(\mathscr{L}_{R}^{\langle k\rangle} ; p\right)$. Consider the Hopf $R$-algebra $R[\mathbf{x} ; p]$. Then $\mathscr{S}^{\langle k\rangle} \simeq$ $R^{\mathbb{N}_{0}^{k}} \simeq R[\mathbf{x} ; p]^{*}$ is an $R$-algebra with multiplication given by the Hurwitz product

$$
*_{p}: \mathscr{S}^{\langle k\rangle} \otimes_{R} \mathscr{S}^{\langle k\rangle} \longrightarrow \mathscr{S}^{\langle k\rangle}, \quad u \otimes v \longmapsto\left[\mathbf{n} \longmapsto \sum_{\mathbf{t} \leq \mathbf{n}}\left(\begin{array}{c}
\mathbf{n} \\
\mathbf{t}
\end{array}\right) u(\mathbf{t}) v(\mathbf{n}-\mathbf{t})\right],
$$

and the unity

$$
\eta_{p}: R \longrightarrow \mathscr{Y}^{\langle k\rangle}, \quad 1_{R} \longmapsto\left[\mathbf{n} \longmapsto \delta_{\mathbf{n}, 0}\right] \quad \text { for every } \mathbf{n} \in \mathbb{N}_{0}^{k} .
$$


By Propositions 5.2 and $5.3,\left(\mathscr{L}_{R}^{\langle k\rangle} ; p\right) \simeq R[\mathbf{x} ; p]^{\circ}$ has the structure of a Hopf $R$-algebra with the coalgebra structure described in Section 5.3, the Hurwitz product (5.16), the unity (5.17), and the antipode

$$
S_{\mathscr{L}\langle k\rangle}: \mathscr{L}^{\langle k\rangle} \longrightarrow \mathscr{L}^{\langle k\rangle}, \quad u \longmapsto\left[\mathbf{i} \longmapsto(-1)^{\mathbf{i}} u(\mathbf{i})\right]
$$

Proposition 5.5 (see [14, Theorem 3]). Let $u$ and $v$ be linearly recursive sequences over $R$ of orders $m, n$ and with characteristic polynomials $f(x), g(x)$, respectively. Then

(1) $u *{ }_{g} v$ is a linearly recursive sequence over $R$ of order $m \cdot n$ and characteristic polynomial $\chi\left(S_{f} \otimes S_{g}\right)$;

(2) $u *_{p} v$ is a linearly recursive sequence over $R$ of order $m \cdot n$ and characteristic polynomial $\chi\left(S_{f} \otimes E_{n}+E_{m} \otimes S_{g}\right)$.

EXAMPLE 5.6. Let $R$ be any ring and let $\left\{x_{n}\right\}_{n=0}^{\infty},\left\{y_{n}\right\}_{n=0}^{\infty} \in \mathscr{Y}_{R}$ be solutions of the difference equations

$$
\begin{aligned}
x_{n+3}-x_{n+2}+x_{n-1}-x_{n} & =0 ; \quad x_{0}=0, x_{1}=1, x_{2}=2 ; \\
y_{n+2}-y_{n+1}+y_{n} & =0 ; \quad y_{0}=1, y_{1}=0 .
\end{aligned}
$$

Then $\left\{x_{n}\right\}_{n=0}^{\infty}$ is a linearly recursive sequence over $R$ with characteristic polynomial $f(x)=x^{3}-x^{2}+x-1$ and $\left\{y_{n}\right\}_{n=0}^{\infty}$ is a linearly recursive sequence over $R$ with characteristic polynomial $g(x)=x^{2}-x+1$.

Notice that

$$
\begin{aligned}
S_{f} \otimes S_{g} & =\left[\begin{array}{ccc}
0 & 0 & 1 \\
1 & 0 & -1 \\
0 & 1 & 1
\end{array}\right] \otimes\left[\begin{array}{cc}
0 & -1 \\
1 & 1
\end{array}\right] \\
& =\left[\begin{array}{cccccc}
0 & 0 & 0 & 0 & 0 & -1 \\
0 & 0 & 0 & 0 & 1 & 1 \\
0 & -1 & 0 & 0 & 0 & 1 \\
1 & 1 & 0 & 0 & -1 & -1 \\
0 & 0 & 0 & -1 & 0 & -1 \\
0 & 0 & 1 & 1 & 1 & 1
\end{array}\right] .
\end{aligned}
$$

Hence, $\left\{z_{n}\right\}_{n=0}^{\infty}:=\left\{x_{n}\right\}_{n=0}^{\infty} * g\left\{y_{n}\right\}_{n=0}^{\infty}$ is by Proposition 5.5 a linearly recursive sequence over $R$ with characteristic polynomial

$$
\chi\left(S_{f} \otimes S_{g}\right)=x^{6}-x^{5}+x^{3}-x+1,
$$

that is, $\left\{z_{n}\right\}_{n=0}^{\infty}$ is a solution of the difference equation

$$
z_{n+6}-z_{n+5}+z_{n+3}-z_{n+1}+z_{n}=0 \quad \text { with initial vector }(0,0,-2,-1,0,1) .
$$

Table 5.1 gives the first 11 terms of the sequence $\left\{z_{n}\right\}_{n=0}^{\infty}$. 
TABLE 5.1

\begin{tabular}{r|rrrrrrrrrrr}
\hline$n$ & 0 & 1 & 2 & 3 & 4 & 5 & 6 & 7 & 8 & 9 & 10 \\
\hline$x_{n}$ & 0 & 1 & 2 & 1 & 0 & 1 & 2 & 1 & 0 & 1 & 2 \\
$y_{n}$ & 1 & 0 & -1 & -1 & 0 & 1 & 1 & 0 & -1 & -1 & 0 \\
$z_{n}$ & 0 & 0 & -2 & -1 & 0 & 1 & 2 & 0 & 0 & -1 & 0 \\
\hline
\end{tabular}

EXAMPLE 5.7. Consider the sequences $\left\{x_{n}\right\}_{n=0}^{\infty}$ and $\left\{y_{n}\right\}_{n=0}^{\infty}$ of Example 5.6. Then

$$
S_{f} \otimes E_{2}+E_{3} \otimes S_{g}=\left[\begin{array}{cccccc}
0 & -1 & 0 & 0 & 1 & 0 \\
1 & 1 & 0 & 0 & 0 & 1 \\
1 & 0 & 0 & -1 & -1 & 0 \\
0 & 1 & 1 & 1 & 0 & -1 \\
0 & 0 & 1 & 0 & 1 & -1 \\
0 & 0 & 0 & 1 & 1 & 2
\end{array}\right] .
$$

By Proposition 5.5, $\left\{z_{n}\right\}_{n=0}^{\infty}=\left\{x_{n}\right\}_{n=0}^{\infty} *_{p}\left\{y_{n}\right\}_{n=0}^{\infty}:=\left\{\sum_{j=0}^{n}\left(\begin{array}{c}n \\ j\end{array}\right) x_{j} \cdot y_{n-j}\right\}_{n=0}^{\infty}$ is a linearly recursive sequence over $R$ with characteristic polynomial

$$
\chi\left(S_{f} \otimes E_{2}+E_{3} \otimes S_{g}\right)=x^{6}-5 x^{5}+14 x^{4}-25 x^{3}+28 x^{2}-15 x+3 .
$$

Hence, $\left\{z_{n}\right\}_{n=0}^{\infty}$ is a solution of the difference equation

$$
z_{n+6}-5 z_{n+5}+14 z_{n+4}-25 z_{n+3}+28 z_{n+2}-15 z_{n+1}+3 z_{n}=0
$$

with initial vector $(0,1,2,-2,-16,-29)$.

Table 5.2 gives the first 9 terms of the sequence $\left\{z_{n}\right\}_{n=0}^{\infty}$.

TABLE 5.2

\begin{tabular}{r|rrrrrrrrr}
\hline$n$ & 0 & 1 & 2 & 3 & 4 & 5 & 6 & 7 & 8 \\
\hline$x_{n}$ & 0 & 1 & 2 & 1 & 0 & 1 & 2 & 1 & 0 \\
$y_{n}$ & 1 & 0 & -1 & -1 & 0 & 1 & 1 & 0 & -1 \\
$z_{n}$ & 0 & 1 & 2 & -2 & -16 & -29 & -12 & 29 & 0 \\
\hline
\end{tabular}

5.6. Cofree comodules. Let $C$ be an $R$-coalgebra. A right $C$-comodule $\left(M, \varrho_{M}\right)$ is called cofree if there exists an $R$-module $K$ such that $\left(M, \varrho_{M}\right) \simeq\left(K \otimes_{R} C, i d_{K} \otimes \Delta_{C}\right)$ as right $C$-comodules. Note that if $K \simeq R^{(\Lambda)}$, a free $R$-module, then $M \simeq R^{(\Lambda)} \otimes_{R} C \simeq C^{(\Lambda)}$ as right $C$-comodules (this is one reason for the terminology cofree).

As a direct consequence of Lemma 4.6, we get the following corollary.

COROLLARY 5.8. Let $M$ be an $R[\mathbf{x}]$-module. Then there are isomorphisms of $R[\mathbf{x}]^{\circ}$ comodules

$$
\mathscr{L}_{M^{*}}^{\langle k\rangle} \simeq M[\mathbf{x}]^{\circ} \simeq M^{*} \otimes_{R} R[\mathbf{x}]^{\circ} \simeq M^{*} \otimes_{R} \mathscr{L}_{R}^{\langle k\rangle}
$$

In particular, $M[\mathbf{x}]^{\circ}\left(\mathscr{L}_{M^{*}}^{\langle k\rangle}\right)$ is a cofree $R[\mathbf{x}]^{\circ}$-comodule $\left(\mathscr{L}_{R}^{\langle k\rangle}\right.$-comodule). 
6. Linearly (bi)recursive bisequences. In this section, we consider the linearly (bi)recursive $k$-bisequences and the reversible $k$-sequences over $R$-modules, where $R$ is an arbitrary commutative ground ring. We generalize the results of $[16,17]$ concerning the bialgebra structure of the linearly recursive sequences over a base field to the case of arbitrary Artinian ground rings.

6.1. Let $M$ be an $R$-module, $\mathbf{l}=\left(l_{1}, \ldots, l_{k}\right) \in \mathbb{N}_{0}^{k}$, and consider the system of linear bidifference equations (SLBE):

$$
\begin{gathered}
x_{\mathbf{z}+\left(l_{1}, 0, \ldots, 0\right)}+\sum_{i=1}^{l_{1}} p_{\left(1, l_{1}-i\right)}(\mathbf{z}) x_{\mathbf{z}+\left(l_{1}-i, 0, \ldots, 0\right)}=g_{1}(\mathbf{z}), \\
x_{\mathbf{z}+\left(0, l_{2}, 0, \ldots, 0\right)+}+\sum_{i=1}^{l_{2}} p_{\left(2, l_{2}-i\right)}(\mathbf{z}) x_{\mathbf{z}+\left(0, l_{2}-i, 0, \ldots, 0\right)}=g_{2}(\mathbf{z}), \\
\vdots \\
x_{\mathbf{z}+\left(0, \ldots, 0, l_{k}\right)}+\sum_{i=1}^{l_{k}} p_{\left(k, l_{k}-i\right)}(\mathbf{z}) x_{\mathbf{z}+\left(0, \ldots, 0, l_{k}-i\right)}=g_{k}(\mathbf{z}),
\end{gathered}
$$

where the $p_{j l}$ 's are $R$-valued functions and the $g_{j}$ 's are $M$-valued functions defined for all $\mathbf{z} \in \mathbb{Z}^{\langle k\rangle}$. If the $g_{j}$ 's are identically zero, then (6.1) is said to be a homogenous SLBE. If the $p_{j l}$ 's are constants, then (6.1) is said to be an SLBE with constant coefficients.

6.2. Bisequences. For an $R$-module $M$ and $k \geq 0$, let

$$
\tilde{\mathscr{S}}_{M}^{\langle k\rangle}:=\left\{\tilde{v}: \mathbb{Z}^{k} \longrightarrow M\right\} \simeq M^{\mathbb{Z}^{k}}
$$

be the $R$-module of $k$-bisequences over $M$. If $M$ (resp., $k$ ) is not mentioned, then we mean $M=R$ (resp., $k=1$ ). For $\widetilde{w} \in \tilde{\mathscr{Y}}_{M}^{\langle k\rangle}$ and $f(\mathbf{x})=\sum_{\mathbf{i}} a_{\mathbf{i}} \mathbf{x}^{\mathbf{i}} \in R\left[\mathbf{x}, \mathbf{x}^{-1}\right]$, define

$$
f(\mathbf{x})-\widetilde{w}=\tilde{v} \in \tilde{\mathscr{T}}_{M}^{\langle k\rangle}, \quad \text { where } \tilde{v}(\mathbf{z}):=\sum_{\mathbf{i}} a_{\mathbf{i}} \widetilde{w}(\mathbf{z}+\mathbf{i}) \forall \mathbf{z} \in \mathbb{Z}^{k}
$$

With this action, $\tilde{\mathscr{S}}_{M}^{\langle k\rangle}$ becomes an $R\left[\mathbf{x}, \mathbf{x}^{-1}\right]$-module. For subsets $I \subset R\left[\mathbf{x}, \mathbf{x}^{-1}\right]$ and $Y \subset$ $\tilde{\mathscr{S}}_{M}^{\langle k\rangle}$, consider

$$
\begin{aligned}
\operatorname{An}_{\widetilde{\mathscr{S}}_{M}^{\langle k\rangle}}(I) & =\left\{\widetilde{w} \in \widetilde{\mathscr{S}}_{M}^{\langle k\rangle} \mid g-\widetilde{w}=0 \text { for every } g \in I\right\}, \\
\operatorname{An}_{R\left[\mathbf{x}, \mathbf{x}^{-1}\right]}(Y) & =\left\{h \in R\left[\mathbf{x}, \mathbf{x}^{-1}\right] \mid h-\tilde{v}=0 \text { for every } \tilde{v} \in Y\right\} .
\end{aligned}
$$

Obviously, $\operatorname{An}_{\tilde{\mathscr{S}}_{M}^{\langle k\rangle}}(I) \subset \tilde{\mathscr{T}}_{M}^{\langle k\rangle}$ is an $R\left[\mathbf{x}, \mathbf{x}^{-1}\right]$-submodule and $\operatorname{An}_{R\left[\mathbf{x}, \mathbf{x}^{-1}\right]}(Y) \triangleleft R\left[\mathbf{x}, \mathbf{x}^{-1}\right]$ is an ideal.

DEFINITION 6.1. Let $M$ be an $R$-module. We call $\widetilde{w} \in \tilde{\mathscr{S}}_{M}^{\langle k\rangle}$ a linearly recursive $k$ bisequence (resp., a linearly birecursive $k$-bisequence) if $\mathrm{An}_{R[\mathrm{x}]}(\widetilde{w})$ is a monic ideal (resp., a reversible ideal). Note that a $k$-bisequence $\tilde{u} \in \tilde{\mathscr{S}}_{M}^{\langle k\rangle}$ is linearly recursive if and only if it is a solution of a homogenous SLBE with constant coefficients of the form (6.1). The subsets $\widetilde{\mathscr{L}}_{M}^{\langle k\rangle} \subseteq \widetilde{\mathscr{S}}_{M}^{\langle k\rangle}$ of linearly recursive $k$-bisequences and $\widetilde{\mathscr{P}}_{M}^{\langle k\rangle} \subseteq \widetilde{\mathscr{T}}_{M}^{\langle k\rangle}$ of linearly birecursive $k$-bisequences over $M$ are obviously $R\left[\mathbf{x}, \mathbf{x}^{-1}\right]$-submodules. 


\section{Reversible sequences over modules}

7.1. Let $M$ be an $R$-module. A $k$-bisequence $\tilde{u}$ is said to be a reverse of $u \in \mathscr{Y}_{M}^{\langle k\rangle}$ if $\left.\tilde{u}\right|_{\mathbb{N}_{0}^{k}}=u$ and $\operatorname{An}_{R[\mathrm{x}]}(\tilde{u})=\operatorname{An}_{R[\mathrm{x}]}(u)$. A linearly recursive $k$-sequence $u$ will be called reversible if $u$ has a reverse $\tilde{u} \in \tilde{\mathscr{L}}_{M}^{\langle k\rangle}$. With $\mathscr{R}_{M}^{\langle k\rangle} \subset \mathscr{L}_{M}^{\langle k\rangle}$, we denote the $R[\mathbf{x}]$-submodule of reversible $k$-sequences over $M$.

LEMMA 7.1 (cf. [16, Proposition 14.11]). Let $R$ be Artinian.

(1) Every monic ideal $I \triangleleft R[\mathbf{x}]$ contains a subset of monic polynomials

$$
\left\{x_{j}^{d_{j}} q_{j}\left(x_{j}\right) \mid q_{j}\left(x_{j}\right) \text { is reversible for } j=1, \ldots, k\right\} \text {. }
$$

(2) Let $M$ be an $R$-module. Then every linearly recursive $k$-bisequence over $M$ is linearly birecursive (i.e., $\widetilde{\mathscr{S}}_{M}^{\langle k\rangle}=\tilde{\mathscr{L}}_{M}^{\langle k\rangle}$ ).

Proof. (1) By [5, Theorem 8.7] every commutative Artinian ring is (up to isomorphism) a direct sum of local Artinian rings. Without loss of generality, let $R$ be a local Artinian ring. The Jacobson radical of $R$,

$$
J(R)=\{r \in R \mid r \text { is not invertible in } R\},
$$

is nilpotent, hence there exists a positive integer $n$ such that $J(R)^{n}=0$. Let $I$ be a monic ideal with a subset of monic polynomials $\left\{g_{1}\left(x_{1}\right), \ldots, g_{k}\left(x_{k}\right)\right\} \subset I$. If $g_{j}\left(x_{j}\right) \equiv$ $f_{j}\left(x_{j}\right)\left(\bmod J(R)\left[x_{j}\right]\right)$ for $j=1, \ldots, k$, then $g_{j}\left(x_{j}\right) \mid f_{j}\left(x_{j}\right)^{n}$, where $n$ is the index of nilpotency of the ideal $J(R)$. Hence, $f_{j}\left(x_{j}\right)^{n} \in I$. If we write $f_{j}\left(x_{j}\right)^{n}=x_{j}^{d_{j}} q_{j}\left(x_{j}\right)$ with $\left(x_{j}, q_{j}\left(x_{j}\right)\right)=1$, then $q_{j}(0) \in U(R)$, that is, $q_{j}\left(x_{j}\right)$ is a reversible polynomial for $j=$ $1, \ldots, k$.

(2) Let $\tilde{u}$ be a linearly recursive $k$-bisequence over $M$. If $R$ is Artinian, then by (1) $\operatorname{An}_{R[\mathbf{x}]}(\tilde{u})$ contains a subset of monic polynomials $\left\{x_{j}^{d_{j}} q_{j}\left(x_{j}\right) \mid q_{j}\left(x_{j}\right)\right.$ is reversible for $j=1, \ldots, k\}$. Then for every $\mathbf{z} \in \mathbb{Z}^{k}$, we have $\left(q_{j}\left(x_{j}\right)-\tilde{u}\right)\left(z_{1}, \ldots, z_{j}, \ldots, z_{k}\right)=\left(x_{j}^{d_{j}} q_{j}\left(x_{j}\right)\right.$ $\rightarrow \tilde{u})\left(z_{1}, \ldots, z_{j}-d_{j}, \ldots, z_{k}\right)=0$. Hence, $\left\{q_{j}\left(x_{j}\right) \mid i=1, \ldots, k\right\} \subset \operatorname{An}_{R[\mathbf{x}]}(\tilde{u})$, that is, $\operatorname{An}_{R[\mathbf{x}]}(\tilde{u})$ is a reversible ideal.

7.2. Backsolving. Let $M$ be an $R$-module. Let $u$ be a linearly recursive sequence over $M$ and assume that $\operatorname{An}_{R[x]}(u)$ contains some monic polynomial of the form $x^{d} q(x)=$ $x^{d}\left(a_{0}+a_{1} x+\cdots+a_{l-1} x^{l-1}+x^{l}\right), a_{0} \in U(R)$. Then

$$
a_{0} u(j+d)+a_{1} u(j+d+1)+\cdots+a_{l-1} u(j+d+l-1)+u(j+d+l)=0 \quad \forall j \geq 0
$$

and we get by backsolving a unique linearly birecursive bisequence $\tilde{u} \in \operatorname{An}_{\tilde{\Im}_{M}}(q(x))$ with $\tilde{u}(n)=u(n)$ for all $n \geq d$. In case $l=0$, The bisequence $\tilde{u} \equiv 0$ and is given for $l \neq 0$ by

$$
\tilde{u}(z):= \begin{cases}u(z), & z \geq d, \\ -a_{0}^{-1}\left(a_{1} \tilde{u}(z+1)+\cdots+a_{l-1} \tilde{u}(z+l-1)+\tilde{u}(z+l)\right), & z<d .\end{cases}
$$

If there are two bisequences $\tilde{v}, \widetilde{w} \in \operatorname{An}_{\tilde{\mathscr{S}}_{M}}(q(x))$ with $\tilde{v}(n)=u(n)=\widetilde{w}(n)$ for all $n \geq d$, then one can easily show by backsolving using $q(x)$ that $\widetilde{v}=\widetilde{w}$. Moreover, we claim that 
$\operatorname{An}_{R[x]}(\tilde{u})=\operatorname{An}_{R[x]}(u)$. It is obvious that $\operatorname{An}_{R[x]}(\tilde{u}) \subseteq \operatorname{An}_{R[x]}(u)$. On the other hand, assume that $g(x)=\sum_{j=0}^{m} b_{j} x^{j} \in \operatorname{An}_{R[x]}(u)$. We prove by induction that $(g-\tilde{u})(z)=0$ for all $z \in \mathbb{Z}$. First of all, note that for all $z \geq d$, we have $(g-\tilde{u})(z)=(g \rightarrow u)(z)=0$. Now, let $z_{0}<d$ and assume that $(g-\tilde{u})(z)=0$ for $z \in\left\{z_{0}, z_{0}+1, \ldots, z_{0}+l-1\right\} \subseteq \mathbb{Z}$. Then we have, for $z=z_{0}-1$,

$$
\begin{aligned}
(g-\tilde{u})\left(z_{0}-1\right) & =\sum_{j=0}^{m} b_{j} \tilde{u}\left(j+z_{0}-1\right) \\
& =\sum_{j=0}^{m} b_{j}\left(\sum_{i=1}^{l}-a_{0}^{-1} a_{i} \tilde{u}\left(j+z_{0}-1+i\right)\right) \\
& =-\sum_{i=1}^{l} a_{0}^{-1} a_{i} \sum_{j=0}^{m} b_{j} \tilde{u}\left(j+z_{0}-1+i\right) \\
& =-\sum_{i=1}^{l} a_{0}^{-1} a_{i}(g-\tilde{u})\left(z_{0}-1+i\right)=0 .
\end{aligned}
$$

If $u$ is a linearly recursive $k$-sequence over $M$ with $k>1$ and $\operatorname{An}_{R[\mathbf{x}]}(u)$ contains a set of monic polynomials $\left\{x_{j}^{d_{j}} q_{j}\left(x_{j}\right) \mid q_{j}\right.$ is reversible for $\left.j=1, \ldots, k\right\}$, then we get by backsolving through $q_{j}\left(x_{j}\right)$ along the $j$ th row for $j=1, \ldots, k$ a unique linearly birecursive $k$-bisequence $\tilde{u} \in \operatorname{An}_{\tilde{y}_{M}^{k j}}\left(q_{1}\left(x_{1}\right), \ldots, q_{k}\left(x_{k}\right)\right)$ with $\tilde{u}(\mathbf{n})=u(\mathbf{n})$ for all $\mathbf{n} \geq \mathbf{d}$ and it follows moreover that $\operatorname{An}_{R[\mathrm{x}]}(\tilde{u})=\operatorname{An}_{R[\mathbf{x}]}(u)$.

LEMMA 7.2. Let $M$ be an $R$-module.

(1) Every birecursive $k$-sequence over $M$ is reversible with unique reverse (which we denote by $\operatorname{Rev}(u)$ ). Moreover, $\mathscr{B}_{M}^{\langle k\rangle}$ becomes a structure of an $R\left[\mathbf{x}, \mathbf{x}^{-1}\right]$-module through $f-u:=\left.(f-\operatorname{Rev}(u))\right|_{\mathbb{N}_{0}^{k}}$.

(2) If $R$ is Artinian, then every reversible $k$-sequence over $M$ is birecursive as well (i.e., $\left.\mathscr{B}_{M}^{\langle k\rangle}=\mathscr{R}_{M}^{\langle k\rangle}\right)$.

Proof. (1) If $u \in \mathscr{B}_{M}^{\langle k\rangle}$, then $\operatorname{An}_{R[\mathbf{x}]}(u)$ contains a set of reversible polynomials $\left\{q_{j}\left(x_{j}\right) \mid j=1, \ldots, k\right\}$ and we get by backsolving (see Section 7.2) a unique linearly birecursive $k$-bisequence $\tilde{u} \in \operatorname{An}_{\tilde{y}_{M}^{\prime k\rangle}}\left(q_{1}\left(x_{1}\right), \ldots, q_{k}\left(x_{k}\right)\right)$ with $\tilde{u}(\mathbf{n})=u(\mathbf{n})$ for all $\mathbf{n} \in \mathbb{N}_{0}^{k}$. For the bisequence $\tilde{u}$, we have as shown above $\operatorname{An}_{R[\mathrm{x}]}(\tilde{u})=\operatorname{An}_{R[\mathrm{x}]}(u)$, that is, $\tilde{u}$ is a reverse of $u$. The last statement is obvious.

(2) By (1), $\mathscr{P}_{M}^{\langle k\rangle} \subseteq \mathscr{R}_{M}^{\langle k\rangle}$. If $R$ is Artinian and $u \in \mathscr{R}_{M}^{\langle k\rangle}$ with reverse $\tilde{u}$, then $\operatorname{An}_{R[\mathbf{x}]}(u)=$ $\operatorname{An}_{R[\mathbf{x}]}(\tilde{u})$ is, by Lemma 7.1(2), reversible, that is, $u \in \mathscr{B}_{M}^{\langle k\rangle}$.

EXAMPLE 7.3. The Fibonacci sequence $\digamma=(0,1,1,2,3,5, \ldots)$ has elementary characteristic polynomial $f(x)=x^{2}-x-1$. Since $f(0)=-1$ is invertible in $\mathbb{Z}$, we conclude that $\digamma$ is reversible with reverse

$$
\operatorname{Rev}(\digamma)(z)= \begin{cases}\digamma(z), & z \geq 0 \\ \operatorname{Rev}(\digamma)(z+2)-\operatorname{Rev}(\digamma)(z+1), & z<0\end{cases}
$$

Table 7.1 lists some of the terms of the bisequence $\operatorname{Rev}(\digamma) \in \operatorname{An}_{\tilde{S}_{\mathbb{Z}}}\left(x^{2}-x-1\right)$. 
TABLE 7.1

\begin{tabular}{c|rrrrrrrrr}
\hline$z$ & -4 & -3 & -2 & -1 & 0 & 1 & 2 & 3 & 4 \\
\hline $\operatorname{Rev}(\digamma)(z)$ & -3 & 2 & -1 & 1 & 0 & 1 & 1 & 2 & 3 \\
\hline
\end{tabular}

LEMMA 7.4. There is an isomorphism of $R\left[\mathbf{x}, \mathbf{x}^{-1}\right]$-modules

$$
\widetilde{\mathscr{B}}_{M}^{\langle k\rangle} \simeq \mathscr{S}_{M}^{\langle k\rangle} .
$$

Proof. By Lemma 7.2, we have the well-defined $R\left[\mathbf{x}, \mathbf{x}^{-1}\right]$-linear map

$$
\operatorname{Rev}(-): \mathscr{B}_{M}^{\langle k\rangle} \longrightarrow \widetilde{\mathscr{S}}_{M}^{\langle k\rangle}, \quad u \longmapsto \operatorname{Rev}(u),
$$

where $\operatorname{Rev}(u)$ is the linearly birecursive sequence defined in (7.4).

It is easy to see that $\operatorname{Rev}(-)$ is bijective with inverse $\left.\tilde{u} \mapsto \tilde{u}\right|_{\mathbb{N}_{0}^{k}}$.

7.3. Let $M$ be an $R$-module. We call a $k$-sequence $u \in \mathscr{Y}_{M}^{\langle k\rangle}$ periodic (resp., degenerating) if $\mathbf{x}^{\mathbf{d}}\left(\mathbf{x}^{\mathbf{t}}-u\right)=0$ for some $\mathbf{d} \in \mathbb{N}_{0}^{k}$ and $\mathbf{t} \in \mathbb{N}^{k}$ (resp., $\mathbf{x}^{\mathbf{d}}-u=0$ for some $\mathbf{d} \in \mathbb{N}_{0}^{k}$ ). It is clear that the subsets $\mathscr{P}_{M}^{\langle k\rangle} \subseteq \mathscr{L}_{M}^{\langle k\rangle}$ of periodic $k$-sequences and $\mathscr{D}_{M}^{\langle k\rangle} \subseteq \mathscr{L}_{M}^{\langle k\rangle}$ of degenerating $k$-sequences are $R[\mathbf{x}]$-submodules.

REMARK 7.5 (see [16, Proposition 5.2]). If $M$ is a finite $R$-module, then every linearly recursive sequence over $M$ is periodic (i.e., $\mathscr{P}_{M}^{\langle 1\rangle}=\mathscr{L}_{M}^{\langle 1\rangle}$ ).

Proposition 7.6 (see [16, Proposition 5.27]). Let $R$ be an arbitrary commutative ring, $M$ an $R$-module, and denote by $\mathscr{R} \mathscr{P}_{M}^{\langle k\rangle}$ the set of reversible periodic $k$-sequences over $M$. Then there is an isomorphism of $R[\mathbf{x}]$-modules

$$
\mathscr{P}_{M}^{\langle k\rangle} \simeq \mathscr{D}_{M}^{\langle k\rangle} \oplus \mathscr{R} \mathscr{P}_{M}^{\langle k\rangle} .
$$

The following result generalizes Proposition 7.6 and describes the $R[\mathbf{x}]$-module structure of arbitrary linearly recursive $k$-sequences of $R$-modules, where $R$ is an $A r$ tinian commutative ground ring.

Proposition 7.7. Let $M$ be an $R$-module. If $R$ is Artinian, then there are isomorphisms of $R[\mathbf{x}]$-modules

$$
\mathscr{L}_{M}^{\langle k\rangle} \simeq \mathscr{D}_{M}^{\langle k\rangle} \oplus \tilde{\mathscr{L}}_{M}^{\langle k\rangle}=\mathscr{D}_{M}^{\langle k\rangle} \oplus \tilde{\mathscr{B}}_{M}^{\langle k\rangle} \simeq \mathscr{D}_{M}^{\langle k\rangle} \oplus \mathscr{P}_{M}^{\langle k\rangle}=\mathscr{D}_{M}^{\langle k\rangle} \oplus \mathscr{R}_{M}^{\langle k\rangle} .
$$

Proof. If $u$ is a linearly recursive sequence over $M$, then $\operatorname{An}_{R[\mathrm{x}]}(u)$ contains, by Lemma 7.1(1), a set of monic polynomials $\left\{x^{d_{j}} \mathcal{q}_{j}\left(x_{j}\right) \mid \mathcal{q}_{j}\left(x_{j}\right)\right.$ is reversible for $j=$ $1, \ldots, k\}$. By backsolving (see Section 7.2), we have a well-defined morphism of $R[\mathbf{x}]$ modules

$$
\gamma: \mathscr{L}_{M}^{\langle k\rangle} \longrightarrow \tilde{\mathscr{L}}_{M}^{\langle k\rangle}, \quad u \longmapsto \tilde{u},
$$

where $\tilde{u}$ is the unique linearly birecursive bisequence $\tilde{u} \in \operatorname{An}_{\widetilde{s}_{M}}\left(q_{1}, \ldots, q_{k}\right)$ with $\tilde{u}(\mathbf{n})=$ $u(\mathbf{n})$ for all $\mathbf{n} \geq \mathbf{d}$. It is clear that $\operatorname{Ke}(\gamma)=\mathscr{D}_{M}^{\langle k\rangle}$. On the other hand, there is a morphism 
of $R[\mathbf{x}]$-modules

$$
\beta=\tilde{\mathscr{L}}_{M}^{\langle k\rangle} \longrightarrow \mathscr{L}_{M}^{\langle k\rangle}, \quad \widetilde{w} \longmapsto \widetilde{w}_{\left.\right|_{\mathbb{N}_{0}^{k}} ^{k}}
$$

It is obvious that $\gamma \circ \beta=i d_{\widetilde{\mathscr{x}}_{M}^{\langle k\rangle}}$, hence the exact sequence

$$
0 \longrightarrow \mathscr{D}_{M}^{\langle k\rangle} \longrightarrow \mathscr{L}_{M}^{\langle k\rangle} \stackrel{\gamma}{\longrightarrow} \tilde{\mathscr{L}}_{M}^{\langle k\rangle} \longrightarrow 0,
$$

of $R[\mathbf{x}]$-modules splits, that is, $\mathscr{L}_{M}^{\langle k\rangle} \simeq \mathscr{D}_{M}^{\langle k\rangle} \oplus \tilde{\mathscr{L}}_{M}^{\langle k\rangle}$. Since $R$ is Artinian, we have by Lemmata 7.1(2) and 7.2(2) $\tilde{\mathscr{L}}_{M}^{\langle k\rangle}=\tilde{\mathscr{S}}_{M}^{\langle k\rangle}$ and $\mathscr{S}_{M}^{\langle k\rangle}=\mathscr{R}_{M}^{\langle k\rangle}$. We are done now by the isomorphism of $R[\mathbf{x}]$-modules $\mathscr{B}_{M}^{\langle k\rangle} \simeq \widetilde{\mathscr{S}}_{M}^{\langle k\rangle}$ (Lemma 7.4).

7.4. The Hopf $R$-algebra $R\left[\mathbf{x}, \mathbf{x}^{-1}\right]$. Consider the commutative group $G$ generated by $\left\{x_{j} \mid j=1, \ldots, k\right\}$. Then the ring of Laurent polynomials $R\left[\mathbf{x}, \mathbf{x}^{-1}\right]=R G$ has the structure of a commutative cocommutative Hopf $R$-algebra $\left(R\left[\mathbf{x}, \mathbf{x}^{-1}\right], \mu, \eta, \Delta, \varepsilon, S\right)$, where $\mu$ (resp., $\eta$ ) is the usual multiplication (resp., the usual unity) and for all $z \in \mathbb{Z}, j=1, \ldots, k$,

$$
\begin{gathered}
\Delta: R\left[\mathbf{x}, \mathbf{x}^{-1}\right] \longrightarrow R\left[\mathbf{x}, \mathbf{x}^{-1}\right] \otimes_{R} R\left[\mathbf{x}, \mathbf{x}^{-1}\right], \quad x_{j}^{z} \longmapsto x_{j}^{z} \otimes x_{j}^{z}, \\
\varepsilon: R\left[\mathbf{x}, \mathbf{x}^{-1}\right] \longrightarrow R, \quad x_{j}^{z} \longmapsto 1_{R}, \\
S: R\left[\mathbf{x}, \mathbf{x}^{-1}\right] \longrightarrow R\left[\mathbf{x}, \mathbf{x}^{-1}\right], \quad x_{j}^{z} \longmapsto x_{j}^{-z} .
\end{gathered}
$$

Proposition 7.8. Let $R$ be an arbitrary commutative ring. Then $R\left[\mathbf{x}, \mathbf{x}^{-1}\right]$ is an admissible Hopf $R$-algebra and $R\left[\mathbf{x}, \mathbf{x}^{-1}\right]^{\circ}$ is a Hopf $R$-algebra.

Proof. Notice that $R\left[\mathbf{x}, \mathbf{x}^{-1}\right]$ is a cofinitary Hopf $R$-algebra by Lemma 4.6(2). Consider the proof of Proposition 5.2 and replace $R[\mathbf{x}]$ with $R\left[\mathbf{x}, \mathbf{x}^{-1}\right]$. Then the map

$$
T_{j}: B \longrightarrow B, \quad b \longmapsto \bar{\Delta}\left(x_{j}\right) b
$$

is invertible with inverse

$$
\bar{T}_{j}: B \longrightarrow B, \quad b \longmapsto \bar{\Delta}\left(x_{j}^{-1}\right) b .
$$

Then the matrix $M_{j}$ of $T_{j}$ is invertible and $\chi_{j}(0) \in U(R)$ for $j=1, \ldots, k$. Consequently, $\mathscr{K}_{R\left[\mathbf{x}, \mathbf{x}^{-1}\right]}$ satisfies axiom (A1). Since $R\left[\mathbf{x}, \mathbf{x}^{-1}\right] / \operatorname{Ke}(\varepsilon) \simeq R, \mathscr{K}_{R\left[\mathbf{x}, \mathbf{x}^{-1}\right]}$ satisfies axiom (A2). Consider the bijective antipode $S$ of $R\left[\mathbf{x}, \mathbf{x}^{-1}\right]$. For every ideal $I \triangleleft R\left[\mathbf{x}, \mathbf{x}^{-1}\right], S^{-1}(I) \triangleleft$ $R\left[\mathbf{x}, \mathbf{x}^{-1}\right]$ is an ideal and we have an isomorphism of $R$-modules $R\left[\mathbf{x}, \mathbf{x}^{-1}\right] / S^{-1}(I) \simeq$ $R\left[\mathbf{x}, \mathbf{x}^{-1}\right] / I$. Hence, $\mathscr{K}_{R\left[\mathbf{x}, \mathbf{x}^{-1}\right]}$ satisfies axiom (A3). Consequently, $R\left[\mathbf{x}, \mathbf{x}^{-1}\right]$ is an admissible Hopf $R$-algebra. The last statement follows now by Proposition 4.3.

For every $R$-module $M$, we have an isomorphism of $R\left[\mathbf{x}, \mathbf{x}^{-1}\right]$-modules

$$
\Psi_{M}: M\left[\mathbf{x}, \mathbf{x}^{-1}\right]^{*} \longrightarrow \tilde{\mathscr{S}}_{M^{*}}^{\langle k\rangle}, \quad \tilde{\varphi} \longmapsto\left[\mathbf{z} \longmapsto\left[m \longmapsto \tilde{\varphi}\left(m \mathbf{x}^{\mathbf{z}}\right)\right]\right]
$$

with inverse $\tilde{u} \mapsto\left[m \mathbf{x}^{\mathbf{z}} \mapsto \tilde{u}(\mathbf{z})(m)\right]$.

As in the proof of Proposition 5.3, we get the following proposition. 
Proposition 7.9. Let $R$ be an arbitrary ring and $M$ an $R$-module. Then (7.17) induces an isomorphism of $R\left[\mathbf{x}, \mathbf{x}^{-1}\right]$-modules

$$
M\left[\mathbf{x}, \mathbf{x}^{-1}\right]^{\circ} \simeq \widetilde{\mathscr{P}}_{M^{*}}^{\langle k\rangle} .
$$

Proof. Consider the isomorphism of $R\left[\mathbf{x}, \mathbf{x}^{-1}\right]$-modules $M\left[\mathbf{x}, \mathbf{x}^{-1}\right]^{*} \stackrel{\Psi_{M}}{=} \tilde{\mathscr{S}}_{M^{*}}^{\langle k\rangle}$, see (7.17). Let $\varkappa \in M\left[\mathbf{x}, \mathbf{x}^{-1}\right]^{\circ}$. Then $I-\varkappa=0$ for some $R$-cofinite $R\left[\mathbf{x}, \mathbf{x}^{-1}\right]$-ideal $I \triangleleft R\left[\mathbf{x}, \mathbf{x}^{-1}\right]$ and so $I \rightarrow \Psi_{M}(\varkappa)=\Psi_{M}(I-\varkappa)=0$. By Lemma 4.6(2), $I$ is a reversible ideal and so $\operatorname{An}_{R[\mathbf{x}]}(u) \supset I \cap R[\mathbf{x}]$ is a reversible ideal, that is, $\Psi_{M}(\varkappa)$ is linearly birecursive.

On the other hand, let $\tilde{u} \in \widetilde{\mathscr{B}}_{M^{*}}^{\langle k\rangle}$. Then $\operatorname{An}_{R[\mathbf{x}]}(\tilde{u})$ is, by definition, a reversible ideal, that is, it contains a subset of reversible polynomials $\left\{q_{j}\left(x_{j}\right), j=1, \ldots, k\right\}$. Note that for arbitrary $g \in R\left[\mathbf{x}, \mathbf{x}^{-1}\right]$, we have $g q_{j} \rightarrow \Psi_{M}^{-1}(\tilde{u})=\Psi_{M}^{-1}\left(g q_{j} \rightarrow \tilde{u}\right)=\Psi_{M}^{-1}\left(g \rightarrow\left(q_{j} \rightarrow \tilde{u}\right)\right)=$ 0 for $j=1, \ldots, k$. By Lemma $4.6(2)$, the reversible ideal $\left(q_{1}\left(x_{1}\right), \ldots, q_{k}\left(x_{k}\right)\right) \triangleleft R\left[\mathbf{x}, \mathbf{x}^{-1}\right]$ is $R$-cofinite, that is, $\Psi_{M}^{-1}(\tilde{u}) \in M\left[\mathbf{x}, \mathbf{x}^{-1}\right]^{\circ}$.

7.5. The Hopf $R$-algebra structures on $\widetilde{\mathscr{B}}^{\langle k\rangle}$ and $\mathscr{B}^{\langle k\rangle}$. Let $R$ be an arbitrary ring and consider the Hopf $R$-algebra $R\left[\mathbf{x}, \mathbf{x}^{-1}\right]$. Then $\tilde{\mathscr{S}}^{\langle k\rangle} \simeq R^{\mathbb{Z}^{k}} \simeq R\left[\mathbf{x}, \mathbf{x}^{-1}\right]^{*}$ is an $R$-algebra with the Hadamard product

$$
*: \tilde{\mathscr{S}}^{\langle k\rangle} \otimes_{R} \tilde{\mathscr{S}}^{\langle k\rangle} \longrightarrow \tilde{\mathscr{S}}^{\langle k\rangle}, \quad \tilde{u} \otimes \tilde{v} \longmapsto[\mathbf{z} \longmapsto \tilde{u}(\mathbf{z}) \widetilde{v}(\mathbf{z})],
$$

and the unity

$$
\eta: R \longrightarrow \tilde{\mathscr{S}}^{\langle k\rangle}, \quad 1_{R} \longmapsto\left[\mathbf{z} \longmapsto 1_{R}\right] \quad \text { for every } \mathbf{z} \in \mathbb{Z}^{k}
$$

By Proposition $7.8, R\left[\mathbf{x}, \mathbf{x}^{-1}\right]^{\circ}$ is a Hopf $R$-algebra. So $\mathscr{B}^{\langle k\rangle} \simeq R\left[\mathbf{x}, \mathbf{x}^{-1}\right]^{\circ}$ inherits the structure of a Hopf $R$-algebra $\left(\mathscr{B}^{\langle k\rangle}, *_{g}, \eta_{g}, \Delta_{\mathscr{B}}\langle k\rangle, \varepsilon_{\mathscr{\beta}}\langle k\rangle, S_{\mathscr{B}^{\prime}}\langle k\rangle\right)$, where $*_{g}$ is the Hadamard product (5.14), $\eta_{g}$ is the unity (5.15), and

$$
\begin{aligned}
\Delta_{\mathscr{B}}\langle k\rangle & : \mathscr{B}^{\langle k\rangle} \longrightarrow \mathscr{P}^{\langle k\rangle} \otimes_{R} \mathscr{B}^{\langle k\rangle}, & & u \sum_{\mathbf{t} \leq \mathbf{1}-\mathbf{1}}\left(\mathbf{x}^{\mathbf{t}}-u\right) \otimes e_{\mathbf{t}}^{\mathbf{F}}, \\
\varepsilon_{\mathscr{B}}\langle k\rangle & : \mathscr{B}^{\langle k\rangle} \longrightarrow R, & u & \longmapsto u(\mathbf{0}), \\
S_{\mathscr{B}}\langle k\rangle & : \mathscr{B}^{\langle k\rangle} \longrightarrow \mathscr{P}^{\langle k\rangle}, & u & \longmapsto \mathbf{n} \longmapsto \operatorname{Rev}(u)(-\mathbf{n})] .
\end{aligned}
$$

Moreover, $\widetilde{\mathscr{B}}^{\langle k\rangle} \simeq R\left[\mathbf{x}, \mathbf{x}^{-1}\right]^{\circ}$ becomes a Hopf $R$-algebra $\left(\widetilde{\mathscr{B}}^{\langle k\rangle}, *_{g}, \eta_{g}, \Delta_{\widetilde{\mathscr{B}}_{\langle\rightarrow}\langle k\rangle}, \varepsilon_{\widetilde{\Re}_{\mathfrak{B}}\langle k\rangle}, S_{\widetilde{\mathscr{B}}\langle k\rangle}\right)$, where $*$ is the Hadamard product (7.19), $\eta$ is the unity (7.20), and

$$
\begin{aligned}
& \Delta_{\widetilde{\mathscr{B}}_{\langle 0}\langle k\rangle}: \widetilde{\mathscr{S}}^{\langle k\rangle} \longrightarrow \widetilde{\mathscr{B}}^{\langle k\rangle} \otimes_{R} \widetilde{\mathscr{B}}^{\langle k\rangle}, \quad \tilde{u} \longmapsto \sum_{\mathbf{t} \leq \mathbf{l}-\mathbf{1}} \operatorname{Rev}\left(\mathbf{x}^{\mathbf{t}}-\tilde{u}_{\left.\right|_{\mathbb{N}_{0}^{k}}}\right) \otimes \operatorname{Rev}\left(e_{\mathbf{t}}^{\mathbf{F}}\right), \\
& \varepsilon_{\widetilde{\Re}}\langle k\rangle: \widetilde{\mathscr{B}}^{\langle k\rangle} \longrightarrow R, \quad \tilde{u} \longmapsto \tilde{u}(\mathbf{0}), \\
& S_{\widetilde{\mathscr{B}}_{\mathfrak{B}}\langle k\rangle}: \widetilde{\mathscr{B}}^{\langle k\rangle} \longrightarrow \widetilde{\mathscr{S}}^{\langle k\rangle}, \quad \tilde{u} \longmapsto[\mathbf{z} \longmapsto \tilde{u}(-\mathbf{z})] .
\end{aligned}
$$

Note that with these structures the isomorphism $\mathscr{B}^{\langle k\rangle} \simeq \widetilde{\mathscr{B}}^{\langle k\rangle}$ of Lemma 7.4 turns to be an isomorphism of Hopf $R$-algebras.

The following theorem extends the corresponding result from the case of a base field [17, page 124] (see also [16, Proposition 14.15]) to the case of arbitrary Artinian ground rings. 
THEOREM 7.10. If $R$ is Artinian, then there are isomorphisms of $R$-bialgebras

$$
\mathscr{L}^{\langle k\rangle} \simeq \mathscr{D}^{\langle k\rangle} \oplus \tilde{\mathscr{L}}^{\langle k\rangle}=\mathscr{D}^{\langle k\rangle} \oplus \widetilde{\mathscr{B}}^{\langle k\rangle} \simeq \mathscr{D}^{\langle k\rangle} \oplus \mathscr{B}^{\langle k\rangle}=\mathscr{D}^{\langle k\rangle} \oplus \mathscr{R}^{\langle k\rangle}
$$

Proof. Consider the isomorphism $\mathscr{L}^{\langle k\rangle} \simeq \mathscr{D}^{\langle k\rangle} \oplus \tilde{\mathscr{L}}^{\langle k\rangle}$, see (7.10). With the help of Lemmata 4.5 and 7.1, one can show, as in [17, page 123], that $\gamma: \mathscr{L}^{\langle k\rangle} \rightarrow \tilde{\mathscr{L}}^{\langle k\rangle}$, see (7.11), and $\beta: \tilde{\mathscr{L}}^{\langle k\rangle} \rightarrow \mathscr{L}^{\langle k\rangle}$, see (7.12), are in fact bialgebra morphisms. Obviously, $\operatorname{Ke}(\gamma)=$ $\mathscr{D}^{\langle k\rangle} \subset \mathscr{L}^{\langle k\rangle}$ is an $\mathscr{L}^{\langle k\rangle}$-subbialgebra and we are done.

As an analog to Corollary 5.8, we get the following corollary.

COROLLARY 7.11. Let $M$ be an $R\left[\mathbf{x}, \mathbf{x}^{-1}\right]$-module. Then there are isomorphisms of $R\left[\mathbf{X}, \mathbf{X}^{-1}\right]^{\circ}$-comodules

$$
\tilde{\mathscr{L}}_{M^{*}}^{\langle k\rangle} \simeq M\left[\mathbf{x}, \mathbf{x}^{-1}\right]^{\circ} \simeq M^{*} \otimes_{R} R\left[\mathbf{x}, \mathbf{x}^{-1}\right]^{\circ} \simeq M^{*} \otimes_{R} \tilde{\mathscr{L}}_{R}^{\langle k\rangle}
$$

In particular, $M\left[\mathbf{x}, \mathbf{x}^{-1}\right]^{\circ}\left(\tilde{\mathscr{L}}_{M^{*}}^{\langle k\rangle}\right)$ is a cofree $R\left[\mathbf{x}, \mathbf{x}^{-1}\right]^{\circ}$-comodule $\left(\tilde{\mathscr{L}}_{R}^{\langle k\rangle}\right.$-comodule).

As a consequence of [2, Theorem 2.4.7] and [2, Corollaray 2.5.10], we get the following corollary.

COROLLARY 7.12. Let $R$ be Noetherian and consider the $R$-bialgebra $R[\mathbf{x} ; g]^{\circ}$ (resp., the Hopf R-algebra $R[\mathbf{x} ; p]^{\circ}$, the Hopf $R$-algebra $R\left[\mathbf{x}, \mathbf{x}^{-1}\right]^{\circ}$ ). If $A$ is an $\alpha$-algebra (resp., an $\alpha$-bialgebra, a Hopf $\alpha$-algebra), then there are isomorphisms of $R$-coalgebras (resp., $R$-bialgebras, Hopf R-algebras)

$$
\begin{gathered}
A[\mathbf{x} ; g]^{\circ} \simeq A^{\circ} \otimes_{R} R[\mathbf{x} ; g]^{\circ}, \quad A[\mathbf{x} ; p]^{\circ} \simeq A^{\circ} \otimes_{R} R[\mathbf{x} ; p]^{\circ}, \\
A\left[\mathbf{x}, \mathbf{x}^{-1}\right]^{\circ} \simeq A^{\circ} \otimes_{R} R\left[\mathbf{x}, \mathbf{x}^{-1}\right]^{\circ} .
\end{gathered}
$$

7.6. Representative functions. Let $G$ be a monoid (a group) and consider the $R$ algebra $B=R^{G}$ with pointwise multiplication. Then $B$ is an $R G$-bimodule under the left and right actions

$$
(y f)(x)=f(x y), \quad(f y)(x)=f(y x) \quad \forall x, y \in G .
$$

We call $f \in R^{G}$ an $R$-valued representative function on the monoid $G$ if $(R G) f(R G)$ is finitely generated as an $R$-module. If $R$ is Noetherian, then the subset $\mathscr{R}(G) \subset R^{G}$ of all representative functions on $G$ is an $R G$-subbimodule. Moreover, we deduce from [4, Theorem 2.13 and Corollary 2.15] that in case $(R G)^{\circ} \subset R^{G}$ is pure, we have an isomorphism of $R$-bialgebras (Hopf $R$-algebras) $\mathscr{R}(G) \simeq(R G)^{\circ}$.

COROLlarY 7.13. Let $R$ be Noetherian.

(1) Considering the monoid $\left(\mathbb{N}_{0}^{k},+\right)$, there are isomorphisms of $R$-bialgebras

$$
\mathscr{R}\left(\mathbb{N}_{0}^{k}\right) \simeq R[\mathbf{x} ; p]^{\circ} \simeq \mathscr{L}_{R}^{\langle k\rangle}
$$


(2) Considering the group $\left(\mathbb{Z}^{k},+\right)$, there are isomorphisms of Hopf $R$-algebras

$$
\mathscr{R}\left(\mathbb{Z}^{k}\right) \simeq R\left[\mathbf{x}, \mathbf{x}^{-1}\right]^{\circ} \simeq \tilde{\mathscr{P}}_{R}^{\langle k\rangle} \simeq \mathscr{B}_{R}^{\langle k\rangle} .
$$

ACKNOWLEDGMENTS. I am so grateful to my advisor Professor Robert Wisbauer for his wonderful supervision and continuous encouragement and support. The author is also grateful to the referees for helpful suggestions and for drawing his attention to $[12,13]$ by Kurakin.

\section{REFERENCES}

[1] E. Abe, Hopf Algebras, Cambridge Tracts in Mathematics, vol. 74, Cambridge University Press, Cambridge, 1980.

[2] J. Y. Abuhlail, Dualitätstheoreme für Hopf-Algebren über Ringen, Ph.D. Dissertation, Heinrich-Heine Universität, Düsseldorf, Germany, 2001, http://www.ulb.uniduesseldorf.de/diss/mathnat/2001/abuhlail.html.

[3] J. Y. Abuhlail, J. Gómez-Torrecillas, and F. J. Lobillo, Duality and rational modules in Hopf algebras over commutative rings, J. Algebra 240 (2001), no. 1, 165-184.

[4] J. Y. Abuhlail, J. Gómez-Torrecillas, and R. Wisbauer, Dual coalgebras of algebras over commutative rings, J. Pure Appl. Algebra 153 (2000), no. 2, 107-120.

[5] M. F. Atiyah and I. G. Macdonald, Introduction to Commutative Algebra, Addison-Wesley Publishing, Massachusetts, 1969.

[6] W. Chin and J. Goldman, Bialgebras of linearly recursive sequences, Comm. Algebra 21 (1993), no. 11, 3935-3952.

[7] L. Grünenfelder, Über die Struktur von Hopf-Algebren, Ph.D. Dissertation, Eidgenössische Technische Hochschule, Zürich, 1969.

[8] L. Grünenfelder and T. Košir, Koszul cohomology for finite families of comodule maps and applications, Comm. Algebra 25 (1997), no. 2, 459-479.

[9] L. Grünenfelder and M. Omladič, Linearly recursive sequences and operator polynomials, Linear Algebra Appl. 182 (1993), 127-145.

[10] T. Honold and A. Nechaev, Weighted modules and representations of codes, Problems Inform. Transmission 35 (1999), 205-223.

[11] B. W. Jones, Linear Algebra, Holden-Day, San Francisco, 1973.

[12] V. Kurakin, Hopf algebras of linear recurring sequences over commutative rings, Proceedings of the 2nd Mathematical Workshop of MSSU (Moscow, 1994), 1994, pp. 67-69 (Russian).

[13] _ Hopf algebras of linear recurring sequences over rings and modules, preprint, 2000.

[14] _ A Hopf algebra dual to a polynomial algebra over a commutative ring, Mat. Zametki 71 (2002), no. 5, 677-685.

[15] V. L. Kurakin, A. S. Kuzmin, V. T. Markov, A. V. Mikhalev, and A. A. Nechaev, Linear codes and polylinear recurrences over finite rings and modules (a survey), Applied Algebra, Algebraic Algorithms and Error-Correcting Codes (Honolulu, HI, 1999) (M. Fossorier, H. Imai, S. Lin, and A. Poli, eds.), Lecture Notes in Comput. Sci., vol. 1719, SpringerVerlag, Berlin, 1999, pp. 365-391.

[16] V. L. Kurakin, A. S. Kuzmin, A. V. Mikhalev, and A. A. Nechaev, Linear recurring sequences over rings and modules, J. Math. Sci. 76 (1995), no. 6, 2793-2915.

[17] R. G. Larson and E. J. Taft, The algebraic structure of linearly recursive sequences under Hadamard product, Israel J. Math. 72 (1990), no. 1-2, 118-132.

[18] A. V. Mikhalev and A. A. Nechaev, Linear recurring sequences over modules, Acta Appl. Math. 42 (1996), no. 2, 161-202.

[19] S. Montgomery, Hopf Algebras and Their Actions on Rings, CBMS Regional Conference Series in Mathematics, vol. 82, American Mathematical Society, District of Columbia, 1993. 
[20] A. Nechaev, Linear recurrent sequences over quasi-Frobenius modules, Russian Math. Surveys 48 (1993), 209-210.

[21]___ Polylinear recurring sequences over modules and quasi-Frobenius modules, 1st International Tainan-Moscow Algebra Workshop (Tainan, 1994) (Y. Fong, U. Knauer, and A. V. Mikhalev, eds.), de Gruyter, Berlin, 1996, pp. 283-298.

[22] B. Peterson and E. J. Taft, The Hopf algebra of linearly recursive sequences, Aequationes Math. 20 (1980), no. 1, 1-17.

[23] M. E. Sweedler, Hopf Algebras, Mathematics Lecture Note Series, W. A. Benjamin, New York, 1969.

[24] E. J. Taft, Linearly recursive tableaux, Congr. Numer. 107 (1995), 33-36.

Jawad Y. Abuhlail: Mathematics Department, Birzeit University, P.O. Box 14, Birzeit, West Bank, Palestine

Current address: Box \# 281 KFUPM, 31261 Dhaharan, Saudia Arabia

E-mail address: jabuh1ai1@birzeit.edu; abuh1ai1@kfupm.edu.sa 


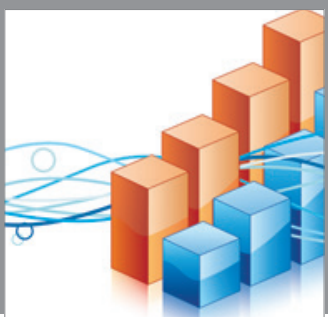

Advances in

Operations Research

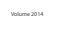

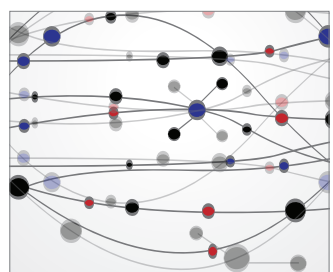

\section{The Scientific} World Journal
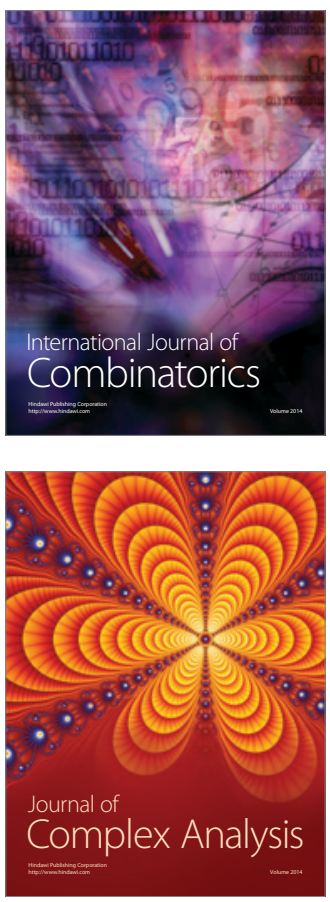

International Journal of

Mathematics and

Mathematical

Sciences
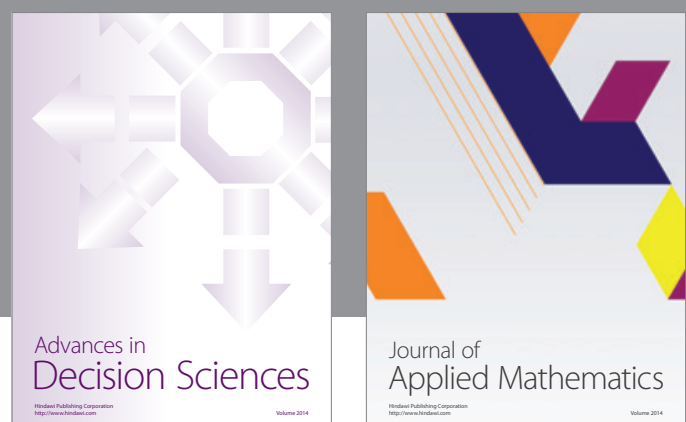

Journal of

Applied Mathematics
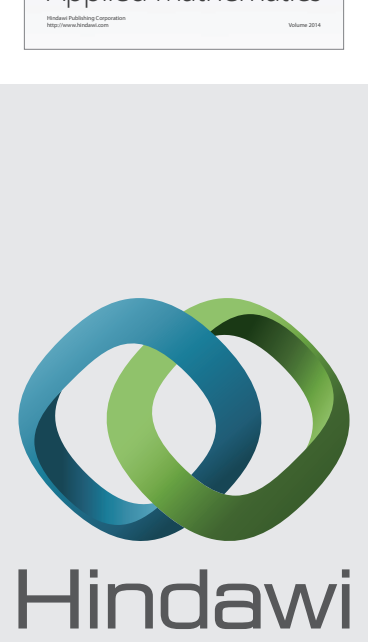

Submit your manuscripts at http://www.hindawi.com
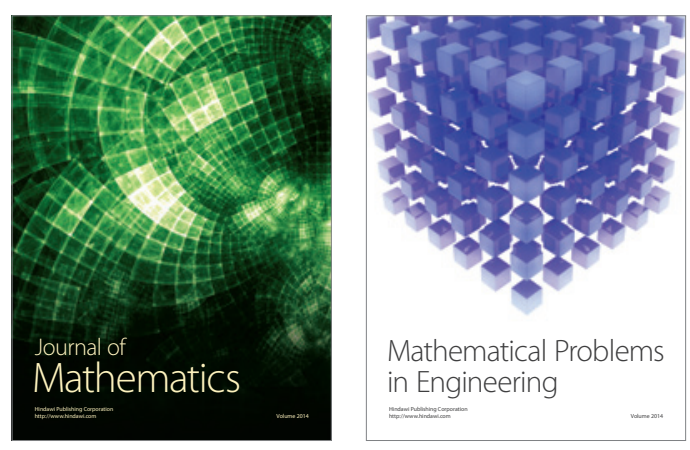

Mathematical Problems in Engineering
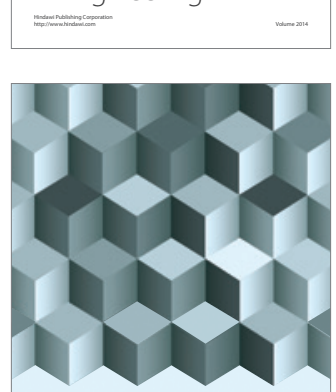

Journal of

Function Spaces
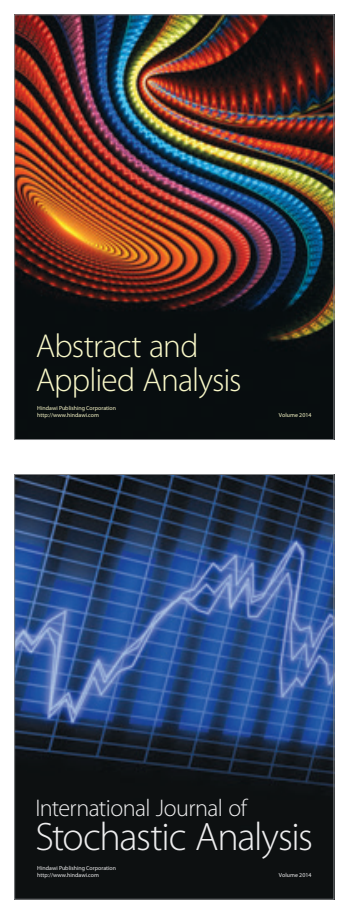

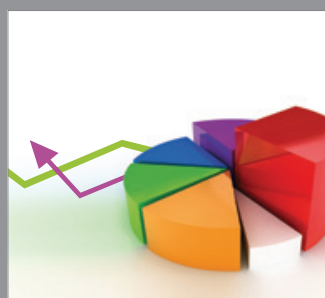

ournal of

Probability and Statistics

Promensencen
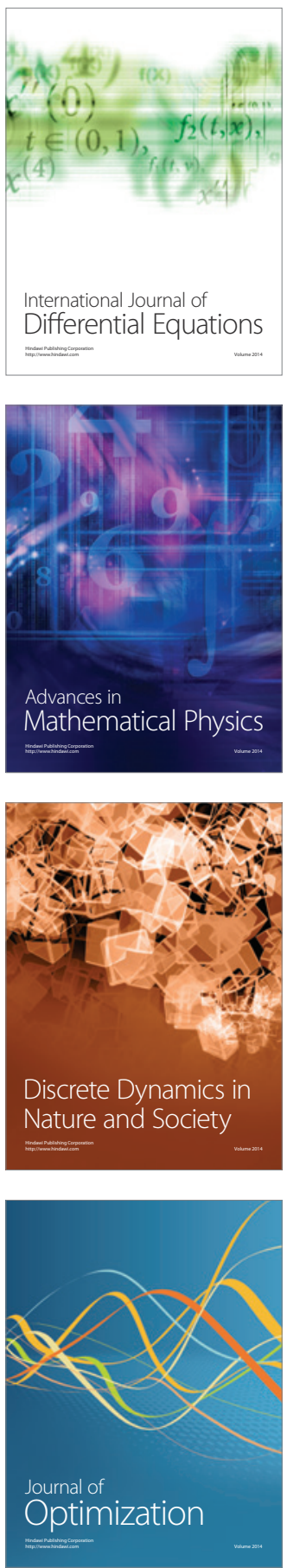